TRANSACTIONS OF THE

AMERICAN MATHEMATICAL SOCIETY

Volume 361, Number 10, October 2009, Pages 5193-5221

S 0002-9947(09)04879-X

Article electronically published on April 30, 2009

\title{
EXCITED YOUNG DIAGRAMS AND EQUIVARIANT SCHUBERT CALCULUS
}

\author{
TAKESHI IKEDA AND HIROSHI NARUSE
}

\begin{abstract}
We describe the torus-equivariant cohomology ring of isotropic Grassmannians by using a localization map to the torus fixed points. We present two types of formulas for equivariant Schubert classes of these homogeneous spaces. The first formula involves combinatorial objects which we call "excited Young diagrams", and the second one is written in terms of factorial Schur $Q$ - or $P$-functions. As an application, we give a Giambelli-type formula for the equivariant Schubert classes. We also give combinatorial and Pfaffian formulas for the multiplicity of a singular point in a Schubert variety.
\end{abstract}

\section{INTRODUCTION}

In this paper, we give explicit descriptions of the Schubert classes in the (torus) equivariant cohomology ring of the Grassmannians as well as the maximal isotropic Grassmannians of both symplectic and orthogonal types. Our main results express the image of an equivariant Schubert class under the localization map to the torus fixed points.

Now let us fix some notation. Let $G$ be a complex semisimple connected algebraic group. Choose a maximal torus $T$ of $G$ and a Borel subgroup $B$ containing $T$. Let $P$ be a maximal parabolic subgroup of $G$ containing $B$. We are interested in the (integral) $T$-equivariant cohomology ring $H_{T}^{*}(G / P)$ of the homogeneous space $G / P$. The equivariant Schubert classes are parametrized by the set $W^{P}$ of minimal length representatives for $W / W_{P}$, where $W$ is the Weyl group of $G$ and $W_{P}$ is the parabolic subgroup associated to $P$. The set $W^{P}$ also parametrizes the $T$-fixed points $(G / P)^{T}$ in $G / P$. In fact if we put $e_{v}=v P\left(v \in W^{P}\right)$, then $(G / P)^{T}=\left\{e_{v}\right\}_{v \in W^{P}}$. Let $B_{-}$ denote the opposite Borel subgroup such that $B_{-} \cap B=T$. Define the Schubert variety $X_{w}$ associated to the element $w \in W^{P}$ to be the closure of $B_{-}$-orbit $B_{-} e_{w}$ of $e_{w}$. Note that the codimension of $X_{w}$ in $G / P$ is $\ell(w)$, the length of $w$, and $e_{v} \in X_{w}$ if and only if $w \leq v$, where $\leq$ is the partial order on $W^{P}$ induced by the Bruhat-Chevalley ordering of $W$. Since $X_{w}$ is a $T$-stable subvariety in $G / P$, it induces a $T$-equivariant fundamental class, the equivariant Schubert class, denoted by $\left[X_{w}\right] \in H_{T}^{2 \ell(w)}(G / P)$. Our main goal is to describe $\left[X_{w}\right]$ explicitly.

In this paper, we consider $G / P$ in the following list:

- Type $A_{n-1}: S L(n) / P_{d}(1 \leq d \leq n-1)$,

- Type $B_{n}: S O(2 n+1) / P_{n}$,

- Type $C_{n}: S p(2 n) / P_{n}$,

- Type $D_{n}: S O(2 n) / P_{d}(d=n-1, n)$,

Received by the editors September 4, 2007.

2000 Mathematics Subject Classification. Primary 05E15; Secondary 14N15, 14M15, 05E05.

(C)2009 American Mathematical Society Reverts to public domain 28 years from publication 
where we denote by $P_{d}$ the maximal parabolic subgroup associated to the $d$-th simple root (the simple roots being indexed as in [6]). It is well known that the space $S L(n) / P_{d}$ can be identified with the Grassmannian $\mathcal{G}_{d, n}$ of $d$-dimensional subspaces in $\mathbb{C}^{n}$. Any other $G / P$ in the above list is a maximal isotropic Grassmannian with respect to an orthogonal or symplectic form (see Section 6.4 for details).

Our description is based on the ring homomorphism

$$
\iota^{*}: H_{T}^{*}(G / P) \longrightarrow H_{T}^{*}\left((G / P)^{T}\right)=\bigoplus_{v \in W^{P}} H_{T}^{*}\left(e_{v}\right)
$$

induced by the inclusion $\iota:(G / P)^{T} \hookrightarrow G / P$. This $\iota^{*}$ is known to be injective and called the localization map. Each summand $H_{T}^{*}\left(e_{v}\right)$ is canonically isomorphic to the symmetric algebra $S=\operatorname{Sym}_{\mathbb{Z}}(\hat{T})$ of the character group $\hat{T}$ of the torus $T$. Thus the equivariant Schubert class $\left[X_{w}\right]$ is described by a list $\left\{\left.\left[X_{w}\right]\right|_{v}\right\}_{v \in W^{P}}$ of polynomials in $S$, where $\left.\left[X_{w}\right]\right|_{v}$ denote the image of the equivariant Schubert class $\left[X_{w}\right]$ under the homomorphism $\iota_{v}^{*}: H_{T}^{*}(G / P) \longrightarrow H_{T}^{*}\left(e_{v}\right)$ induced by the inclusion $\iota_{v}:\left\{e_{v}\right\} \hookrightarrow G / P$.

In the type $A_{n-1}$ case (cf. Section 5, Theorem 5.4), Knutson and Tao 15] discovered that $\left.\left[X_{w}\right]\right|_{v}$ can be identified with a suitably specialized 'factorial' Schur function, a multi-parameter deformation of a Schur function (see Section 5 for the definition). Their argument uses a remarkable vanishing property of the factorial Schur function (cf. Proposition 5.1). By a totally different method, Lakshmibai, Raghavan, and Sankaran 22 showed the same result, although they did not state it explicitly in terms of the factorial Schur function. In fact, they started with a combinatorial expression for $\left.\left[X_{w}\right]\right|_{v}$ in terms of a set of non-intersecting paths, which came from a detailed analysis of the Gröbner basis of the defining ideal of the Schubert variety due to Kreiman and Lakshmibai [19, and Kodiyalam and Raghavan [12, and then rewrote the expression into a ratio of some determinants, which is a form of a factorial Schur function.

Type $C_{n}$, the case of a Lagrangian Grassmannian, was studied in a paper [10] by the first named author, where $\left.\left[X_{w}\right]\right|_{v}$ is expressed in terms of factorial Schur $Q$-function defined by Ivanov [11. The proof is a comparison of Pieri-Chevalleytype recurrence relations for both $\left.\left[X_{w}\right]\right|_{v}$ and the factorial Schur $Q$-function. This strategy of identification goes well for other $G / P$ in our list above. Actually, we prove in this paper the analogous result for types $B_{n}$ and $D_{n}$, the orthogonal Grassmannian; i.e., we present a formula for $\left.\left[X_{w}\right]\right|_{v}$ in terms of a factorial Schur $P$-function for these spaces (Section 3, Theorem 8.7).

As an application of these formulas, we obtained a Giambelli-type formula (Corollary 8.8) for isotropic Grassmannians that expresses an arbitrary equivariant Schubert class as a Pfaffian of Schubert classes associated with the 'two-row' (strict) partitions. This formula is an equivariant analogue of the Giambelli formula due to Pragacz [26] in the case of ordinary cohomology.

Another type of formula (Section 3, Theorem 3.2. Section 6, Theorem 6.4) that we discuss in the present paper involves combinatorial objects, which we call excited Young diagrams (EYDs for short). The idea of EYDs was inspired by the work 22] of Lakshmibai, Raghavan, and Sankaran mentioned above. These formulas have a 'positive' nature in the sense that it is expressed as a sum over a set of EYDs with each summand being a product of some positive roots. 
For an exposition, here we consider $\mathcal{G}_{d, n}$. It is well known that the set $W^{P_{d}}$ is parametrized by the set of partitions $\lambda=\left(\lambda_{1}, \ldots, \lambda_{d}\right)$ such that $n-d \geq \lambda_{1} \geq$ $\cdots \geq \lambda_{d} \geq 0$, or equivalently the Young diagrams contained in the rectangle of shape $d \times(n-d)$. Let us denote by $D_{\lambda}$ the Young diagram of $\lambda$. Suppose $w, v$ are elements of $W^{P_{d}}$ such that $e_{v} \in X_{w}$. Let $\lambda, \mu$ be the corresponding partitions for $w, v$ respectively. Then we have $D_{\lambda} \subset D_{\mu}$. Our formula expresses $\left.\left[X_{w}\right]\right|_{v}$ as a weighted sum over a set $\mathcal{E}_{\mu}(\lambda)$ (see Subsection 3.4 for the definition) of subsets of $D_{\mu}$. For example, let $\lambda=(3,2), \mu=(4,4,3,1)$. Some typical elements in $\mathcal{E}_{\mu}(\lambda)$ are illustrated below. Here we depict the Young diagrams in Russian style.

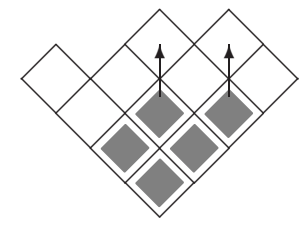

Ground state Two boxes can be excited

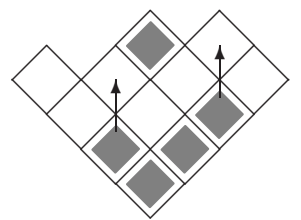

Excited state

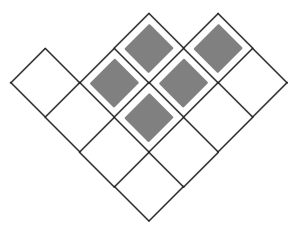

No box can be excited

Let us imagine that each diagram labels a 'quantum state'. Each box can be excited (i.e., raised) to the space one unit north if the neighbors in north, northeast, and northwest directions are all unoccupied (see Subsection 3.4 for the precise definition of excitation). In this case, there are nine excited states obtainable by applying successive excitations starting from the ground state, i.e., the element $D_{\lambda}$.

It should be mentioned that the notion of EYDs and its shifted analogue were introduced by Kreiman 17, 18 independently. Kreiman 17 proved that the set of non-intersecting paths that appeared in [19, 12, 22 is naturally bijective to the set of EYDs. He also presented a combinatorial formula of $\left.\left[X_{w}\right]\right|_{v}$ for the type $C_{n}$ case in terms of the shifted analogue of EYDs by using a result by Ghorpade and Raghavan [8] analogous to [19], 12. In this paper, we present a different proof for these results without using the Gröbner machinery mentioned above. An advantage of our method is that we can apply the same argument to isotropic Grassmannians of orthogonal type, for which no explicit description of the Gröbner basis is known. In order to deal with the case of 'even' orthogonal Grassmannian, we introduce another variant of shifted EYDs.

Our method begins by identifying the localized classes $\left.\left[X_{w}\right]\right|_{v}$ as $\xi$-functions defined by Kostant and Kumar ([16]). Then we can make use of a well-known formula (Proposition 2.3. cf. 4], 1]) that expresses an arbitrary $\xi$-function as a sum over a set of 'reduced subwords', and then use a theory by Stembridge [30 on fully commutative elements in Coxeter groups. The theory enables us to establish a natural bijection between the set of reduced subwords appearing in the sum formula and a certain set of EYDs. This bijection is the technical heart of our proof of Theorem 3.2 and Theorem 6.4.

It is known that the multiplicity $m_{v}\left(X_{w}\right)$ at $e_{v}$ in $X_{w}$ is closely related to $\left.\left[X_{w}\right]\right|_{v}$. Such a multiplicity has been studied in detail by many authors (see [5]). By our combinatorial formula, we can express $m_{v}\left(X_{w}\right)$ as the number of elements in a certain set of excited Young diagrams. Also, we can obtain a closed formula for $m_{v}\left(X_{w}\right)$, which is a specialization of a factorial Schur function. This leads to a Pfaffian formula for the multiplicity of a singular point in a Schubert variety. 
In Section 2, we explain the relation between the polynomial $\left.\left[X_{w}\right]\right|_{v}$ and the $\xi$-function. Some fundamental properties of $\xi$-functions are presented for later use.

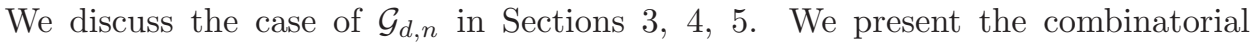
formula in Section 3, and the proof is given in Section 4. We give the closed formula in Section 5, which can be read independently from the preceding two sections. The isotropic Grassmannians are treated in a parallel manner in Sections 6. 7 8. In Section 9 we discuss some application for $m_{v}\left(X_{w}\right)$. In Section 10 we discuss a relation between two types of formulas (Theorems 6.4 and 8.7) by using a Gessel-Viennot-type argument.

\section{Note added.}

(1) After the first version of the present paper was finished, a preprint 28] by Raghavan and Upadhyay appeared. They compute the multiplicity, or more generally the Hilbert function, of the local ring of a Schubert variety at torus fixed points for the type $D_{n}$ case. In particular, they proved a combinatorial formula for the multiplicity equivalent to ours (Corollary 9.3 (9.3)). Their approach is based on standard monomial theory and is quite different from ours.

(2) Combinatorics used in 3, 7, 13, 14 are closely connected with EYDs. In fact "excitation" corresponds to possible moves in a diagram called an RC-graph (or reduced pipe dreams). To any permutation, there is a double Schubert polynomial of Lascoux and Schützenberger (24). In [14 the double Schubert polynomial associated with "vexillary" permutations, a class of permutations including the Grassmannian permutations, are expressed as a sum over "flagged tableaux." Combined with this result and a wellknown fact that the double Schubert polynomials indexed by Grassmannian permutations are factorial Schur functions, one has a combinatorial formula for $\left.\left[X_{w}\right]\right|_{v}$ (in type $A$ ) quite similar to the one in Theorem 3.2, but they are different formulas at least in the sense that the cardinalities of the summands of both formulas are different in general.

Future problems. As an application of Theorem 8.7 one can give a ring presentation for $H_{T}^{*}(G / P)$ in types $B$ and $D$ (see [10] for the type $C$ case). We will discuss the details in a separate paper. These descriptions should be related to results in [15] on degeneracy loci.

The results on multiplicity can be extended to co-minuscule $G / P$ 's. We will discuss the subject elsewhere.

\section{2. $\xi$-FunCtions of Kostant and Kumar}

In this section we introduce the family of functions $\xi^{w}$ for $w \in W$ defined by Kostant and Kumar. By virtue of the result of Arabia 2, we can identify $\xi^{w}\left(w \in W^{P}\right)$ with the equivariant Schubert class $\left[X_{w}\right]$ in $H_{T}^{*}(G / P)$.

Let $R^{+}$denote the set of positive roots with respect to $B$. For $\beta \in R_{+}$, we denote by $\beta^{\vee}$ its dual coroot and $s_{\beta} \in W$ the reflection corresponding to $\beta$. Let $\left\{\alpha_{1}, \ldots, \alpha_{r}\right\}$ be the set of simple roots in $R^{+}$. The Weyl group $W$ is a Coxeter group generated by the set of simple reflections $\left\{s_{1}, \ldots, s_{r}\right\}$, where $s_{i}=s_{\alpha_{i}}$. In particular, we can talk of the length $\ell(w)$ of any element $w \in W$. For a simple reflection $\alpha$ we denote by $\varpi_{\alpha}$ the corresponding fundamental weight. 
Proposition 2.1 (Kostant and Kumar [16]). There exist a family of functions $\xi^{w}: W \longrightarrow S$ for $w \in W$ with the following properties:

(1) $\xi^{w}(v)$ equals zero unless $w \leq v$,

(2) $\xi^{w}(w)=\prod_{\alpha \in R_{+} \cap w R_{-}} \alpha$,

(3) $\xi^{e}(v)=1$ for all $v \in W$, where $e$ is the identity of $W$,

(4) if $\alpha$ is a simple root, then for all $v \in W$,

$$
\xi^{s_{\alpha}}(v)=\varpi_{\alpha}-v\left(\varpi_{\alpha}\right),
$$

(5) if $\alpha$ is a simple root, then

$$
\left(\xi^{s_{\alpha}}-\xi^{s_{\alpha}}(w)\right) \xi^{w}=\sum_{w \stackrel{\beta}{\rightarrow} w^{\prime}}\left\langle w\left(\varpi_{\alpha}\right), \beta^{\vee}\right\rangle \xi^{w^{\prime}}
$$

where we use the notation $w \stackrel{\beta}{\rightarrow} w^{\prime}$ to indicate $w^{\prime}=s_{\beta} w$ for some $\beta \in R_{+}$ and $\ell\left(w^{\prime}\right)=\ell(w)+1$,

(6) each $\xi^{w}(v)$ with $v \in W$ is homogeneous of degree $\ell(w)$.

Remark. We use notation for $\xi$-functions in Kumar's book, which is different from the one used in [16].

The function $\xi^{w}$ is directly related to the object of our main interest.

Proposition 2.2. Let $w \in W^{P}$. We have

$$
\left.\left[X_{w}\right]\right|_{v}=\xi^{w}(v)
$$

for $v \in W^{P}$.

Proof. Arabia 2 proved the result for the flag variety $G / B$ (see Graham's paper 9] for more information). For the parabolic case, the reader can consult Kumar's book 21].

Let $P=P_{d}$ denote the maximal parabolic subgroup associated to $\alpha_{d}$. Note that we have for $w \in W^{P}$

$$
\left(\xi^{s_{d}}-\xi^{s_{d}}(w)\right) \xi^{w}=\sum_{w^{\prime} \in W^{P}, w \stackrel{\beta}{\rightarrow} w^{\prime}}\left\langle w\left(\varpi_{\alpha}\right), \beta^{\vee}\right\rangle \xi^{w^{\prime}} .
$$

The above relation involves only $\xi^{w}\left(w \in W^{P}\right)$. This reflects the fact that the Schubert classes $\left[X_{w}\right]\left(w \in W^{P}\right)$ form an $S$-basis of $H_{T}^{*}(G / P)$, considered a sub$S$-algebra of $H_{T}^{*}(G / B)$ via the projection $G / B \rightarrow G / P$. Note that if $w \in W^{P}$, $\xi^{w}$ is $W_{P}$-invariant in the sense that

$$
\xi^{w}(v u)=\xi^{w}(v) \text { for all } u \in W_{P} .
$$

We can make use of the following formula:

Proposition 2.3 (1], 4]). Let $w, v \in W$ such that $w \leq v$. Fix a reduced expression $s_{i_{1}} \cdots s_{i_{k}}$ for $v$. Put

$$
\beta_{t}=s_{i_{1}} \cdots s_{i_{t-1}}\left(\alpha_{i_{t}}\right) \quad \text { for } \quad 1 \leq t \leq k .
$$

Then

$$
\xi^{w}(v)=\sum_{j_{1}, \ldots, j_{s}} \beta_{j_{1}} \cdots \beta_{j_{s}},
$$

where the sum is over all sequences $1 \leq j_{1}<\cdots<j_{s} \leq k$ such that $s_{i_{j_{1}}} \cdots s_{i_{j_{s}}}$ is a reduced expression for $w$. 
Although the above formula is explicit, it still requires many calculations to get a concrete expression for $\xi^{w}(v)$ in general. If $w$ is an element of $W^{P}$ for classical $G$ and $P$ in our list (cf. Section 1), then we can give a nice combinatorial interpretation for the right hand side of (2.4).

\section{Excited Young Diagrams}

We fix positive integers $n, d$ such that $1 \leq d \leq n$. In this section, we give a combinatorial formula (Theorem 3.2) for the restriction of the equivariant Schubert classes in the Grassmannian $\mathcal{G}_{d, n}$ to any torus fixed points.

3.1. Schubert variety of $\mathcal{G}_{d, n}$. Let $w \in S_{n}$ be a Grassmannian permutation, i.e.,

$$
w(1)<\cdots<w(d), \quad w(d+1)<\cdots<w(n) .
$$

When we identify the space $S L(n) / P_{d}$ with the Grassmannian $\mathcal{G}_{d, n}$ of $d$-dimensional subspaces of $\mathbb{C}^{n}$, the Schubert variety associated with $w$ is given by

$$
X_{w}=\left\{V \in \mathcal{G}_{d, n} \mid \operatorname{dim}\left(V \cap F_{n-w(d-i+1)+1}\right) \geq i \text { for } 1 \leq i \leq d\right\},
$$

where $F_{i}=\operatorname{span}_{\mathbb{C}}\left\{\boldsymbol{e}_{n-i+1}, \ldots, \boldsymbol{e}_{n}\right\}$ is the $i$-plane spanned by the last $i$ vectors in the standard $T$-basis $\boldsymbol{e}_{1}, \ldots, \boldsymbol{e}_{n}$ of $\mathbb{C}^{n}$.

3.2. Partitions and Young diagrams. Let $\lambda=\left(\lambda_{1} \geq \cdots \geq \lambda_{r} \geq 0\right)$ be a partition. To every partition $\lambda$ one associates its Young diagram $D_{\lambda}$ which is the set of square boxes with coordinate $(i, j) \in \mathbb{Z}^{2}$ such that $1 \leq j \leq \lambda_{i}$ :

$$
D_{\lambda}=\left\{(i, j) \in \mathbb{Z}^{2} \mid 1 \leq i \leq r, 1 \leq j \leq \lambda_{i}\right\} .
$$

The boxes in $D_{\lambda}$ are arranged in a plane with matrix-style coordinates. For example,

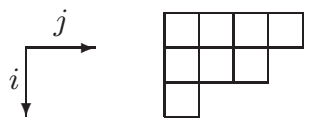

is the Young diagram $D_{\lambda}$ of $\lambda=(4,3,1)$.

Let $\mathcal{P}_{d}$ denote the set of all partitions with length at most $d$. If $n$ is an integer with $n \geq d$, let $\mathcal{P}_{d, n}$ be the subset of $\mathcal{P}_{d}$ consisting of the elements whose largest part is less than or equal to $n-d$. For any partition $\mu$, let $\mathcal{P}_{\mu}$ denote the set of all partitions $\lambda$ such that $\lambda \leq \mu$. In particular, if $\mu$ is the partition whose Young diagram is the $d \times(n-d)$ rectangle, then $\mathcal{P}_{\mu}$ is identical to $\mathcal{P}_{d, n}$.

3.3. Grassmannian permutations and Young diagrams. The set $W^{P_{d}}$ of minimal length coset representatives for $W / W_{P_{d}}$ with $W=S_{n}$ is identified with the set of all Grassmannian permutations. Let $w \in W^{P_{d}}$. We define a partition $\lambda=\left(\lambda_{1}, \ldots, \lambda_{d}\right)$ of $n$ by

$$
\lambda_{j}=w(d-j+1)-d+j-1 \quad(1 \leq j \leq d) .
$$

When considered as a Young diagram, $\lambda$ is contained in the rectangular shape $d \times(n-d)$. Note that we have $\ell(w)=|\lambda|:=\sum_{j=1}^{d} \lambda_{i}$, where $\ell(w)$ is the length of $w$.

There is a convenient way to recover the Grassmannian permutation from a Young diagram as follows. Given a Young diagram $\lambda$ contained in the rectangle $d \times(n-d)$, write a path along the boundary of the Young diagram starting from 
the SW corner to the NE corner of the rectangle. We assign numbers to each arrow from 1 to $n$. For example, for $\lambda=(4,3,1,0)$ with $n=9, d=4$ we have the following picture:

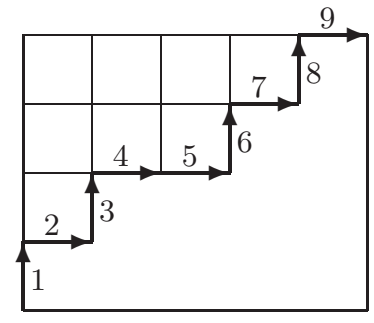

If the assigned numbers of the vertical arrows are $i_{1}<\cdots<i_{d}$ and those of the horizontal arrows are $j_{1}<\cdots<j_{n-d}$, then the corresponding Grassmannian permutation is

$$
w=\left(i_{1}, \ldots, i_{d}, j_{1}, \ldots, j_{n-d}\right) .
$$

Explicitly we have

$$
i_{k}=\lambda_{d-k+1}+k \quad(1 \leq k \leq d), \quad j_{k}=-\lambda_{k}^{\prime}+k+d \quad(1 \leq k \leq n-d),
$$

where $\lambda^{\prime}$ is the conjugate of $\lambda$. In the above example, we have $w=136824579$.

3.4. Excited Young diagrams. Let $\lambda \leq \mu$ be partitions. The Young diagram $D_{\lambda}$ of $\lambda$ is a subset of $D_{\mu}$. Take an arbitrary subset $C$ of $D_{\mu}$. Pick up a box $x \in C$ such that $x+(1,0), x+(0,1), x+(1,1) \in D_{\mu} \backslash C$. Then set $C^{\prime}=C \cup\{x+(1,1)\} \backslash\{x\}$.

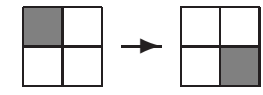

The procedure $C \rightarrow C^{\prime}$ for changing $C$ into $C^{\prime}$ is called an elementary excitation occurring at $x$. If a subset $S$ of $D_{\mu}$ is obtained from $C$ by applying elementary excitations successively, i.e., there is a sequence

$$
C=C_{0} \rightarrow C_{1} \rightarrow \cdots \rightarrow C_{r-1} \rightarrow C_{r}=S, \quad r \geq 0
$$

of elementary excitations, then we say that $S$ is an excited state of $C$, or $S$ is obtained from $C$ by excitation. Let $\mathcal{E}_{\mu}(\lambda)$ denote the set of all excited states of $D_{\lambda}$.

Example 3.1. For example, let $\lambda=(3,1), \mu=(5,4,3)$. Then the set $\mathcal{E}_{\mu}(\lambda)$ consists of the following seven elements:
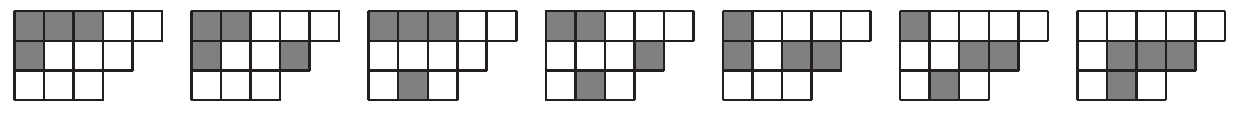

It is easy to see that the number $r$ in (3.1), the number of elementary excitations, is well-defined for $C \in \mathcal{E}_{\mu}(\lambda)$. In fact, if we define the energy $E(C)$ of $C \in \mathcal{E}_{\mu}(\lambda)$ by

$$
E(C)=\sum_{(i, j) \in C} m(i, j)-\sum_{(i, j) \in D_{\lambda}} m(i, j), \quad m(i, j)=\frac{1}{2}(i+j),
$$

then we have $E(C)=r$.

Let $\varepsilon_{1}, \ldots, \varepsilon_{n}$ be a standard basis of the lattice $L=\mathbb{Z}^{n}$. The character group $\hat{T}$ is identified with a sublattice of $L$ spanned by $\varepsilon_{i}-\varepsilon_{i+1}(1 \leq i \leq n-1)$. Now we can state the first combinatorial formula. 
Theorem 3.2 (22], [17]). Let $w \leq v \in W^{P_{d}}$ and $\lambda \leq \mu \in \mathcal{P}_{d, n}$ be the corresponding partitions. Then we have

$$
\left.\left[X_{w}\right]\right|_{v}=\sum_{C \in \mathcal{E}_{\mu}(\lambda)} \prod_{(i, j) \in C}\left(\varepsilon_{v(d+j)}-\varepsilon_{v(d-i+1)}\right) .
$$

The proof of the theorem is given in the next section.

Example 3.3. If $w=124735689, v=157923468 \in W^{P_{d}}$ with $d=4$, then $\lambda=$ $(3,1), \mu=(5,4,3)$. We fill the boxes of $D_{\mu}$ with positive roots as follows:

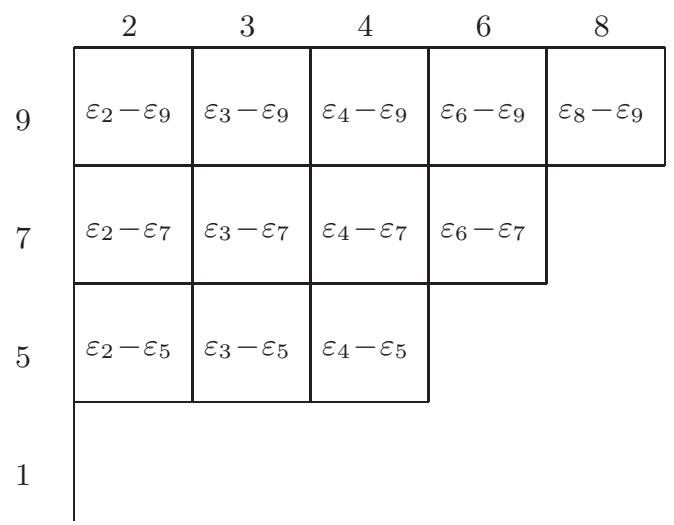

Then our formula reads (cf. Example 3.1):

$$
\begin{aligned}
{\left.\left[X_{w}\right]\right|_{v} } & =\left(\varepsilon_{2}-\varepsilon_{9}\right)\left(\varepsilon_{3}-\varepsilon_{9}\right)\left(\varepsilon_{4}-\varepsilon_{9}\right)\left(\varepsilon_{2}-\varepsilon_{7}\right)+\left(\varepsilon_{2}-\varepsilon_{9}\right)\left(\varepsilon_{3}-\varepsilon_{9}\right)\left(\varepsilon_{6}-\varepsilon_{7}\right)\left(\varepsilon_{2}-\varepsilon_{7}\right) \\
& +\left(\varepsilon_{2}-\varepsilon_{9}\right)\left(\varepsilon_{3}-\varepsilon_{9}\right)\left(\varepsilon_{4}-\varepsilon_{9}\right)\left(\varepsilon_{3}-\varepsilon_{5}\right)+\left(\varepsilon_{2}-\varepsilon_{9}\right)\left(\varepsilon_{3}-\varepsilon_{9}\right)\left(\varepsilon_{6}-\varepsilon_{7}\right)\left(\varepsilon_{3}-\varepsilon_{5}\right) \\
& +\left(\varepsilon_{2}-\varepsilon_{9}\right)\left(\varepsilon_{4}-\varepsilon_{7}\right)\left(\varepsilon_{6}-\varepsilon_{7}\right)\left(\varepsilon_{2}-\varepsilon_{7}\right)+\left(\varepsilon_{2}-\varepsilon_{9}\right)\left(\varepsilon_{4}-\varepsilon_{7}\right)\left(\varepsilon_{6}-\varepsilon_{7}\right)\left(\varepsilon_{3}-\varepsilon_{5}\right) \\
& +\left(\varepsilon_{3}-\varepsilon_{7}\right)\left(\varepsilon_{4}-\varepsilon_{7}\right)\left(\varepsilon_{6}-\varepsilon_{7}\right)\left(\varepsilon_{3}-\varepsilon_{5}\right) .
\end{aligned}
$$

\section{Proof of Theorem 3.2}

4.1. Fully commutative elements. Let $W$ be a Coxeter group. An element $w$ in $W$ is fully commutative if any reduced expression for $w$ can be obtained from any other by using only the Coxeter relations that involve commuting generators. It is known that every element $w$ in $W^{P}$ for every $(G, P)$ in our list (cf. Section 1) is fully commutative ([29], Theorem 6.1).

4.2. Row-reading expression for a Grassmannian permutation. Let $v$ be a Grassmannian permutation in $W^{P_{d}}$ and $\mu \in \mathcal{P}_{d, n}$ be the corresponding partition. To each box $(i, j) \in D_{\mu}$ we fill in the simple reflection $s_{d-i+j}$. For example, let $v=(3571246) \in S_{7}$ with $d=3$. The corresponding partition is $\mu=(4,3,2)$, and we have the following table:

$$
\begin{array}{|l|l|l|l|}
\hline s_{3} & s_{4} & s_{5} & s_{6} \\
\hline s_{2} & s_{3} & s_{4} & \multicolumn{1}{|c}{} \\
\cline { 1 - 2 } s_{1} & s_{2} & \multicolumn{2}{|c}{} \\
\cline { 1 - 2 } & &
\end{array}
$$


We read the entry of the boxes of the Young diagram $D_{\mu}$ from right to left starting from the bottom row to the top row and form a word

$$
s_{i_{1}} \cdots s_{i_{k}} \quad(k=|\mu|),
$$

which gives a reduced expression for $v$. For example we have

$$
v=3571246=s_{2} s_{1} \cdot s_{4} s_{3} s_{2} \cdot s_{6} s_{5} s_{4} s_{3} .
$$

We call the word given by (4.1) the row-reading word of $v$.

The row-reading procedure gives a bijective map

$$
\varphi: D_{\mu} \longrightarrow\{1, \ldots, k\}, \quad k=|\mu| .
$$

For example, if $\mu=(4,3,1)$, then the map $\varphi$ is expressed by the following tableau:

\begin{tabular}{|l|l|l|l|}
\hline 9 & 8 & 7 & 6 \\
\cline { 1 - 2 } 5 & 4 & 3 & \multicolumn{1}{|c}{} \\
\cline { 1 - 2 } 2 & 1 & \multicolumn{2}{|c}{} \\
\cline { 1 - 2 } & &
\end{tabular}

Now let $C$ be an arbitrary subset of $D_{\mu}$ and let $\varphi(C)=\left\{j_{1}, \ldots, j_{r}\right\}$ with $j_{1}<\cdots<$ $j_{r}$ be the image of $\{1, \ldots, k\}$ under the map $\varphi$. Let $s_{i_{j}}$ be the simple reflection assigned to the box $\varphi^{-1}(j)$ in $D_{\mu}$ for $1 \leq j \leq k$. Then we put

$$
w_{C}=s_{i_{j_{1}}} \cdots s_{i_{j_{r}}} \text {. }
$$

For example, if $C$ is the subset of $D_{\mu}$ indicated by the following gray boxes

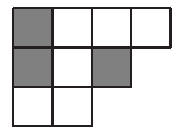

then $w_{C}=s_{4} s_{2} s_{3}$. In particular, if $C=D_{\mu}$, then $w_{C}$ is nothing but the row-reading word of $v$. Given another Grassmannian permutation $w$ such that $w \leq v$, define

$$
\mathcal{R}_{v}(w)=\left\{C \subset D_{\mu} \mid \sharp C=\ell(w), w_{C}=w\right\} .
$$

Note that if $\lambda$ is the partition corresponding to $w$, then $D_{\lambda} \subset D_{\mu}$ and $D_{\lambda} \in \mathcal{R}_{v}(w)$.

Lemma 4.1. Let $C, C^{\prime}$ be subsets of a Young diagram $D_{\mu}$ such that $C^{\prime}$ is obtained from $C$ by an elementary excitation from $C$. If $C$ belongs to $\mathcal{R}_{v}(w)$, then we have $C^{\prime} \in \mathcal{R}_{v}(w)$.

Proof. We may assume that $C^{\prime}$ is obtained from $C$ by an elementary excitation which occurred in the $2 \times 2$-square of the corner $(i, j)$. Set $k:=d+j-i$. Consider the following regions in the diagram $D_{\mu}$ :

$$
\begin{aligned}
R & :=\left\{(i, a) \mid j \leq a \leq \mu_{i}\right\} \cup\{(i+1, b) \mid 1 \leq b \leq j+1\}, \\
R_{\sharp} & :=\left\{(i, a) \mid j+2 \leq a \leq \mu_{i}\right\}, \quad R_{b}:=\{(i+1, b) \mid 1 \leq b \leq j-1\} .
\end{aligned}
$$

If we pick up the $i$-th and the $(i+1)$-th rows, they look like

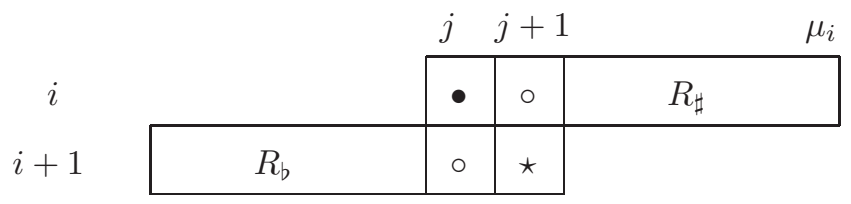


It suffices to compare the subwords corresponding to $C \cap R$ and $C^{\prime} \cap R$ (given by row-reading procedure). In $C$, we have $s_{k}$ at the position $(i, j)$ indicated above by $\bullet$. The positions indicated by $\circ$ are vacant by definition of the elementary excitation. Then $C^{\prime}$ is obtained by moving $s_{k}$ to the position indicated by $\star$. Any simple reflection $s_{l}$ located in $C \cap R_{\sharp}$ (resp. $\left.C \cap R_{b}\right)$ commutes with $s_{k}$ since $l \geq j+2$ (resp. $l \leq j-2$ ). Hence the subword corresponding to the subset $C \cap R$ can be rewritten into the subword corresponding to $C^{\prime} \cap R$ using only the Coxeter relation that involves commuting generators.

Corollary 4.2. We have $\mathcal{E}_{\mu}(\lambda) \subset \mathcal{R}_{v}(w)$.

Proof. Use induction on energy $E(C)$ of $C \in \mathcal{E}_{\mu}(\lambda)$. The corollary is obvious from Lemma 4.1

We would like to establish the following.

Proposition 4.3. We have $\mathcal{E}_{\mu}(\lambda)=\mathcal{R}_{v}(w)$.

In order to prove Proposition 4.3, it suffices to show the following.

Lemma 4.4. Let $C \in \mathcal{R}_{v}(w)$ be such that $C \neq D_{\lambda}$. Then there exists an element $C^{\prime} \in \mathcal{R}_{v}(w)$ such that $C$ is obtained from $C^{\prime}$ by a sequence of elementary excitations.

Proof. Let $s_{i_{j_{1}}} \cdots s_{i_{j_{r}}}$ be the row-reading word for $v$ and $s_{i_{k_{1}}} \cdots s_{i_{k_{r}}}$ be the word corresponding to $C$. Let $a$ be such that $j_{a} \neq k_{a}$ and $j_{t}=k_{t}$ for $a<t \leq r$. We shall compare the two words

$$
s_{i_{k_{1}}} \cdots s_{i_{k_{a}}}, \quad s_{i_{j_{1}}} \cdots s_{i_{j_{a}}} .
$$

Note that the element, say $w^{\prime}$, expressed by the words is fully commutative. Put $t=i_{j_{a}}$. Since $\left\{i_{k_{1}}, \ldots, i_{k_{a}}\right\}$ is equal to $\left\{i_{j_{1}}, \ldots, i_{j_{a}}\right\}$ as a multi-set, we have $t \in$ $\left\{i_{k_{1}}, \ldots, i_{k_{a}}\right\}$. Let $b$ be the largest index such that $i_{k_{b}}=t$. Since $w^{\prime}$ is fully commutative, the simple reflections adjacent to $s_{t}$, i.e., $s_{t+1}, s_{t-1}$, cannot appear in the subword

$$
s_{i_{k_{b+1}}} \cdots s_{i_{k_{a}}} \text {. }
$$

This implies that a region $R$ indicated by the picture below is unoccupied in the diagram $C$ :
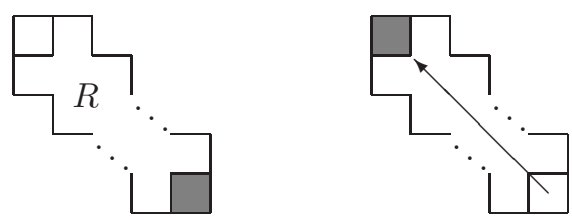

So we can move the box corresponding to $s_{k_{b}}$ as illustrated by the above picture to get a subset $C^{\prime}$ of $D_{\mu}$. Clearly $C^{\prime}$ belongs to $\mathcal{R}_{v}(w)$. Since $C^{\prime}$ has strictly smaller energy than $C$, we see that $C^{\prime}$ belongs to $\mathcal{E}_{\mu}(\lambda)$ by inductive hypothesis. Now by the construction, $C$ is obtained from $C^{\prime}$ by a sequence of elementary excitation. So Lemma 4.1 implies $C$ is also a member of $\mathcal{E}_{\mu}(\lambda)$. 
4.3. $\beta$-sequences. Fix a Grassmannian permutation $v$, and let $\mu \in \mathcal{P}_{d, n}$ be the corresponding partition. Let $\varphi: D_{\mu} \longrightarrow\{1,2, \ldots, k\}$ be the row-reading map of $\mu$. For $(i, j) \in D_{\mu}$ set $\beta_{i, j}=\beta_{\varphi(i, j)}$, where $\beta_{t}(1 \leq t \leq k)$ are defined in (2.3).

Lemma 4.5. For $(i, j) \in D_{\mu}$, we have

$$
\beta_{i, j}=\varepsilon_{v(d+j)}-\varepsilon_{v(d-i+1)} .
$$

Proof. To prove the lemma, we proceed by induction on $|\mu|=\ell(v)$. In the case $\ell(v)=0$, there is nothing to prove. If $\ell(v)>0$, let $v=s_{i_{1}} s_{i_{2}} \cdots s_{i_{\ell}}$ be the rowreading expressions for $v$. Setting $v^{\prime}=s_{i_{2}} \cdots s_{i_{\ell}}$, which is the row-reading expression for $v$, we have $\ell\left(v^{\prime}\right)=\ell(v)-1$. The corresponding shape $\mu^{\prime}$ for $v^{\prime}$ is obtained from $\mu$ by deleting a box of position $\left(r, \mu_{r}\right)$, the leftmost one in the bottom row, where $r$ is the number of rows in $\mu$. Then we have

$$
\beta_{r, \mu_{r}}=\alpha_{i_{1}}=\varepsilon_{d-r+\mu_{r}}-\varepsilon_{d-r+\mu_{r}+1} .
$$

By definition of $\mu_{r}$, we have $\mu_{r}=v(d-r+1)-d+r-1$, so

$$
\beta_{r, \mu_{r}}=\varepsilon_{v(d-r+1)-1}-\varepsilon_{v(d-r+1)} .
$$

Then by Lemma 4.6 below, we have $\beta_{r, \mu_{r}}=\varepsilon_{v\left(d+\mu_{r}\right)}-\varepsilon_{v(d-r+1)}$.

Let $(i, j) \in \mu^{\prime}$. By the hypothesis of induction, we have

$$
\beta_{i, j}^{\prime}=\varepsilon_{v^{\prime}(d+j)}-\varepsilon_{v^{\prime}(d-r+i)}
$$

By definition of $\beta_{i, j}$, it is easy to see that $\beta_{i, j}=s_{i_{1}}\left(\beta_{i, j}^{\prime}\right)$. Hence we have

$$
\beta_{i, j}=s_{i_{1}}\left(\beta_{i, j}^{\prime}\right)=s_{i_{1}}\left(\varepsilon_{v^{\prime}(d+j)}-\varepsilon_{v^{\prime}(d-r+i)}\right)=\varepsilon_{s_{i_{1}} v^{\prime}(d+j)}-\varepsilon_{s_{i_{1}} v^{\prime}(d-r+i)} .
$$

Since $s_{i_{1}} v^{\prime}=v$, we have the lemma.

Lemma 4.6. Let $r$ be the number of rows in $\mu$. Then we have

$$
v(d-r+1)=v\left(d+\mu_{r}\right)+1 .
$$

Proof. For a sequence $a_{1}<a_{2}<\cdots<a_{m}$ of integers, we say $a_{i}$ is a "gap" if $a_{i}-a_{i-1} \geq 2$. Note that $\mu_{r}$ is the largest number such that $v(d+1)<\cdots<v\left(d+\mu_{r}\right)$ has no gap. Since $v$ is a Grassmannian permutation, the number $v\left(d+\mu_{r}\right)+1$ occurs as the smallest gap in $0<v(1)<\cdots<v(d)$. On the other hand, we have $\mu_{d}=\cdots=\mu_{r+1}=0$ and $\mu_{r}>0$, which implies that the smallest gap in $0<v(1)<\cdots<v(d)$ is $v(d-r+1)$.

Proof of Theorem 3.2. By Proposition 2.3 together with Lemma 4.5 we have

$$
\left.\left[X_{w}\right]\right|_{v}=\sum_{C \in \mathcal{R}_{v}(w)} \prod_{(i, j) \in C}\left(\varepsilon_{v(d+j)}-\varepsilon_{v(d-i+1)}\right) .
$$

Then the theorem is immediate from Proposition 4.3

\section{FActorial Schur Functions}

Our main goal in this section is to express $\left.\left[X_{w}\right]\right|_{v}$ for $\mathcal{G}_{d, n}$ as a specialization of a factorial Schur function. First we recall the definition of the factorial Schur functions. 
5.1. Definition of factorial Schur functions. Let $x=\left(x_{1}, \ldots, x_{d}\right)$ be a finite sequence of variables and let $a=\left(a_{i}\right)_{i=1}^{\infty}$ be an infinite sequence of parameters. The factorial Schur function for a partition $\lambda$ of length at most $d$ can be defined as follows. Let

$$
(z \mid a)^{k}=\left(z-a_{1}\right)\left(z-a_{2}\right) \cdots\left(z-a_{k}\right)
$$

for any $k \geq 0$. Then we put

$$
s_{\lambda}^{(d)}(x \mid a)=\frac{\operatorname{det}\left(\left(x_{j} \mid a\right)^{\lambda_{i}+d-i}\right)_{1 \leq i, j \leq d}}{\prod_{1 \leq i<j \leq d}\left(x_{i}-x_{j}\right)} .
$$

This function is actually a polynomial in $x_{1}, \ldots, x_{d}$ and $a_{1}, a_{2}, \ldots, a_{\lambda_{1}+d-1}$, homogeneous of degree $|\lambda|$. In particular we have

$$
s_{1}^{(d)}(x \mid a)=x_{1}+\cdots+x_{d}-a_{1}-\cdots-a_{d} .
$$

For a partition $\lambda \in \mathcal{P}_{d}$, we define a $d$-tuple

$$
a_{\lambda}=\left(a_{\lambda_{d}+1}, a_{\lambda_{d-1}+2}, \ldots, a_{\lambda_{1}+d}\right) \text {. }
$$

Proposition 5.1 (Vanishing property; cf. [25]). We have $s_{\lambda}^{(d)}\left(a_{\mu} \mid a\right)=0$ unless $\mu \geq \lambda$.

Let $\lambda \in \mathcal{P}_{d}$, and take sufficiently large $n$ such that $\lambda$ is contained in the $d \times(n-d)$ rectangle. Define an $n$-tuple $(w(1), \ldots, w(n))$ by

$$
w(i)= \begin{cases}\lambda_{d-i+1}+i & \text { for } 1 \leq i \leq d, \\ -\lambda_{i}^{\prime}+i & \text { for } d<i \leq n\end{cases}
$$

then $w=(w(1), \ldots, w(n))$ is a permutation of $\{1, \ldots, n\}$. If we set $w(i)=i$ for all $i>n$, then the infinite sequence $(w(1), w(2), \ldots)$ does not depend on the choice of $n$.

We also need the following Pieri-type formula:

Lemma 5.2 (cf. 25]). Let $\lambda \in \mathcal{P}_{d}$. We have

$$
\left(s_{1}^{(d)}(x \mid a)-s_{1}^{(d)}\left(a_{\lambda} \mid a\right)\right) s_{\lambda}^{(d)}(x \mid a)=\sum_{\nu} s_{\nu}^{(d)}(x \mid a),
$$

where the summation on the right hand side runs through $\nu \in \mathcal{P}_{d}$ such that $\nu \geq \lambda$ and $|\nu|=|\lambda|+1$.

5.2. Closed formulas. Let $\lambda \in \mathcal{P}_{n, d}$. Recall that $s_{\lambda}^{(d)}(x \mid a)$ is a polynomial in $x_{1}, \ldots, x_{d}$ and $a_{1}, \ldots, a_{n-1}$. A specialization given by $a_{i}=\varepsilon_{i}(1 \leq i \leq n-1)$ is important for our geometric application below. For $v \in W^{P_{d}}$, we define an $n$-tuple by

$$
x_{v}=\left(\varepsilon_{v(1)}, \ldots, \varepsilon_{v(d)}\right) .
$$

Lemma 5.3. Let $v \in W^{P_{d}}$. We have

$$
\left.\left[X_{s_{d}}\right]\right|_{v}=-s_{1}^{(d)}\left(x_{v} \mid \varepsilon_{1}, \ldots, \varepsilon_{n-1}\right) .
$$

Proof. The $d$-th fundamental weight of $S L(n)$ is given by $\varpi_{d}=\varepsilon_{1}+\cdots+\varepsilon_{d}$. By Proposition 2.1 (4) and formula (5.1), the lemma follows.

Theorem 5.4 (Knutson and Tao [15], Lakshmibai, Raghavan, and Sankaran [22]). Let $w \leq v \in W^{P_{d}}$ and $\lambda \leq \mu \in \mathcal{P}_{d, n}$ be the associated partitions. Then we have

$$
\left.\left[X_{w}\right]\right|_{v}=(-1)^{\ell(w)} s_{\lambda}^{(d)}\left(x_{v} \mid \varepsilon_{1}, \ldots, \varepsilon_{n-1}\right) .
$$


Proof. Let $\mathcal{P}_{\mu}$ denote the set of all partitions $\lambda$ such that $\lambda \leq \mu$. Consider the following system of equations for the functions $F_{\lambda}=F_{\lambda}\left(\varepsilon_{1}, \ldots, \varepsilon_{n}\right)\left(\lambda \in \mathcal{P}_{\mu}\right)$ :

$$
\sum_{i=1}^{d}\left(\varepsilon_{\lambda_{d-i+1}+i}-\varepsilon_{\mu_{d-i+1}+i}\right) \cdot F_{\lambda}=\sum_{\nu} F_{\nu} \quad\left(\lambda \in \mathcal{P}_{\mu} \backslash\{\mu\}\right),
$$

where the summation on the right hand side runs through $\nu \in \mathcal{P}_{\mu}$ such that $\nu \geq \lambda$ and $|\nu|=|\lambda|+1$. This equation together with the initial condition $F_{\phi}\left(\varepsilon_{1}, \ldots, \varepsilon_{n}\right)=1$ for the empty partition $\lambda=\phi$ determine the functions $F_{\lambda}\left(\varepsilon_{1}, \ldots, \varepsilon_{n}\right)\left(\lambda \in \mathcal{P}_{\mu}\right)$ uniquely.

Now we can see that equation (2.2) for $\left.\left[X_{w}\right]\right|_{v}$ is identical to (5.3) above, in view of the bijection $W^{P_{d}} \cong \mathcal{P}_{d, n}$. On the other hand, the right hand side of (5.2) satisfies the same equation from Proposition 5.1 and Lemmas 5.2 and 5.3. In addition, both sides of (5.2) satisfy the initial condition. Hence the theorem follows.

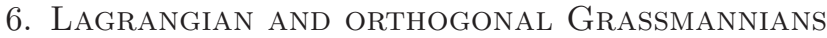

Fix a positive integer $n$. Here we give analogous theorems for other classical homogeneous spaces $G / P$ in the following types:

- Type $B_{n}: G=S O(2 n+1, \mathbb{C}), P=P_{n}$,

- Type $C_{n}: G=S p(2 n, \mathbb{C}), P=P_{n}$,

- Type $D_{n}: G=S O(2 n, \mathbb{C}), P=P_{n-1}, P_{n}$,

where we shall denote by $P_{d}$ the maximal parabolic subgroup associated to the simple root $\alpha_{d}$ (the simple roots being indexed as in [6]).

In type $B_{n}$ (resp. type $C_{n}$ ), the variety $G / P$ can be identified with a closed subvariety of $\mathcal{G}_{n, 2 n+1}\left(\right.$ resp. $\left.\mathcal{G}_{n, 2 n}\right)$ parametrizing the isotropic $n$-spaces in $\mathbb{C}^{2 n+1}$ (resp. $\mathbb{C}^{2 n}$ ) equipped with a non-degenerate symmetric (resp. skew symmetric) form. Our space $G / P$ in type $C_{n}$ is also called the Lagrangian Grassmannian. For the even dimensional space $\mathbb{C}^{2 n}$ equipped with a non-degenerate symmetric form, the isotropic $n$-subspaces constitute a union of two closed subvarieties of $\mathcal{G}_{n, 2 n}$, each of which is isomorphic to $G / P, P$ being one of $P_{n-1}$ and $P_{n}$. Note that the varieties $S O(2 n+1, \mathbb{C}) / P_{n}, S O(2 n+2, \mathbb{C}) / P_{d}(d=n, n+1)$ are all isomorphic (cf. 26] $)$, although they are different as $T$-spaces.

The goal of this section is to present combinatorial formulas for the restriction of equivariant Schubert classes to any torus fixed points for the classical Grassmannians $G / P$.

6.1. The set $W^{P}$. First we fix some notation on a set $W^{P}$ which parametrizes the Schubert classes and the torus fixed points.

The character group $\hat{T}$ of our torus is a lattice with a standard basis $\left\{\varepsilon_{i}\right\}_{i=1}^{r}$, where $r=\operatorname{dim}(T)$.

Type $C_{n}\left(G=S p(2 n, \mathbb{C}), P=P_{n}\right)$ : We identify $W$ with a subgroup of $S_{2 n}$ acting on the functionals $\pm \varepsilon_{1}, \ldots, \pm \varepsilon_{n}$. Denote $\varepsilon_{i},-\varepsilon_{i}$ by $i$ and $\bar{i}$ respectively and define a partial order on the set $I_{n}=\{1, \ldots, n, \bar{n}, \ldots, \overline{1}\}$ by

$$
1<2<\cdots<n<\bar{n}<\cdots<\overline{2}<\overline{1} .
$$

Then we have $W=\left\{w \in S_{2 n} \mid w(\bar{i})=\overline{w(i)}\right\}$, where $a \mapsto \bar{a}$ is the involution on $I_{n}$ given by $\pm \varepsilon_{i} \mapsto \mp \varepsilon_{i}$. Then

$$
W^{P}=\{w \in W \mid w(1)<\cdots<w(n)\} .
$$


The simple roots are

$$
\alpha_{i}=\varepsilon_{i}-\varepsilon_{i+1} \quad(1 \leq i \leq n-1), \quad \alpha_{n}=2 \varepsilon_{n},
$$

and the corresponding simple reflections are

$$
s_{i}=(i, i+1)(\overline{i+1}, \bar{i}) \quad(1 \leq i \leq n-1), \quad s_{n}=(n, \bar{n}) .
$$

We have

$$
\varpi_{n}=\varepsilon_{1}+\cdots+\varepsilon_{n} .
$$

Type $B_{n}\left(G=S O(2 n+1, \mathbb{C}), P=P_{n}\right)$ : Since $W$ is identical to the case $C_{n}$ as a Coxeter group, the description of $W^{P}$ is the same as Type $C_{n}$. The simple roots are given by

$$
\alpha_{i}=\varepsilon_{i}-\varepsilon_{i+1} \quad(1 \leq i \leq n-1), \quad \alpha_{n}=\varepsilon_{n},
$$

and we have

$$
\varpi_{n}=\frac{1}{2}\left(\varepsilon_{1}+\cdots+\varepsilon_{n}\right) .
$$

Type $D_{n+1}\left(G=S O(2 n+2, \mathbb{C}), P=P_{n+1}\right)$ : In this case, we have

$$
W=\left\{w \in S_{2 n+2} \mid w(\bar{i})=\overline{w(i)}, \quad \sharp N(w) \text { is even }\right\},
$$

where we set $N(w)=\{i \mid 1 \leq i \leq n+1, w(i)>n+1\}$. We have

$$
W^{P}=\{w \in W \mid w(1)<\cdots<w(n+1)\} .
$$

The simple reflections are given by

$$
s_{i}=(i, i+1)(\overline{i+1}, \bar{i}) \quad(1 \leq i \leq n), \quad s_{n+1}=(n, \overline{n+1})(n+1, \bar{n}),
$$

and we have

$$
\alpha_{i}=\varepsilon_{i}-\varepsilon_{i+1} \quad(1 \leq i \leq n), \quad \alpha_{n+1}=\varepsilon_{n}+\varepsilon_{n+1},
$$

and

$$
\varpi_{n+1}=\frac{1}{2}\left(\varepsilon_{1}+\cdots+\varepsilon_{n+1}\right) .
$$

Remark 1. For type $D_{n+1}$, the result for $P=P_{n}$ is obtained by simply replacing $\varepsilon_{n+1}$ by $-\varepsilon_{n+1}$. So we only consider $P_{n+1}$.

6.2. Strict partitions and shifted Young diagrams. Next we need a description of the set $W^{P}$ in terms of strict partitions. Let $\lambda=\left(\lambda_{1}>\cdots>\lambda_{r}>0\right)$ be a strict partition. Then the shifted Young diagram of $\lambda$ is the array of boxes with $\lambda_{i}$ boxes in the $i$-th row, such that each row is shifted by one position to the right relative to the preceding row. More explicitly, given a strict partition $\lambda_{1}>\lambda_{2}>\cdots>\lambda_{r}>0$, the shifted diagram of $\lambda$ is defined to be

$$
D_{\lambda}^{\prime}:=\left\{(i, j) \in \mathbb{Z}^{2} \mid 1 \leq i \leq l, i \leq j<\lambda_{i}+i\right\} .
$$

For example

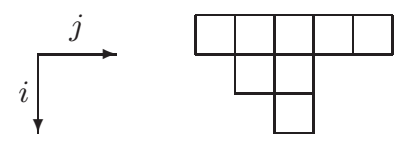

is the shifted Young diagram of $\lambda=(5,2,1)$. 
Define a partial order $\lambda \leq \mu$ if and only if $\lambda_{i} \leq \mu_{i}$ for all $i$. Denote the particular element $\rho_{n}=(n, \ldots, 2,1)$. Let $\mathcal{S P}_{\mu}$ denote the set of all strict partitions $\lambda$ such that $\lambda \leq \mu$. The cardinality of the set $\mathcal{S} \mathcal{P}_{\rho_{n}}$ is $2^{n}$.

Proposition 6.1. Let $G / P$ be as above. There is a natural order-preserving bijection

$$
W^{P} \cong \mathcal{S} \mathcal{P}_{\rho_{n}}
$$

If $\lambda \in \mathcal{S} \mathcal{P}_{\rho_{n}}$ corresponds to $w \in W^{P}$ we have $\ell(w)=|\lambda|$.

Explicitly, the bijections are given as follows:

Types $B_{n}, C_{n}$ : Let $w \in W^{P}$. Since $w$ is a Grassmannian permutation of $2 n$ letters (with $d=n$ ) we have the associated Young diagram, say $\Lambda$. It is symmetric and contained in an $n \times n$ square. Then the associated element $\lambda \in \mathcal{S P}_{\rho_{n}}$ is the upper part of the symmetric Young diagram including the diagonal. Thus if $\Lambda=\left(\Lambda_{1}, \ldots, \Lambda_{n}\right)$, then

$$
\lambda_{i}=\max \left\{\Lambda_{i}-i+1,0\right\} \quad(1 \leq i \leq n) .
$$

Type $D_{n+1}$ : Let $w \in W^{P}$. Then the associated Young diagram, say $\Lambda$, is symmetric and contained in an $(n+1) \times(n+1)$ square. Then the associated element $\lambda \in \mathcal{S P}_{\rho_{n}}$ is the "strictly" upper part of the symmetric Young diagram. Thus if $\Lambda=\left(\Lambda_{1}, \ldots, \Lambda_{n+1}\right)$, then

$$
\lambda_{i}=\max \left\{\Lambda_{i}-i, 0\right\} \quad(1 \leq i \leq n) .
$$

Example 6.2. Take $w_{1}=24 \overline{3} \overline{1}$ for $D_{4}$ and $w_{2}=2 \overline{3} \overline{1}$ for $B_{3}$ (or $C_{3}$ ). Then the associated strict partitions are the same and given by $\lambda=(3,1)$.

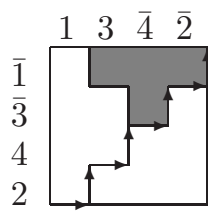

$D_{4} \quad B_{3}, C_{3}$

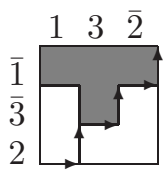

$$
B_{3}, C_{3}
$$

Note that $w_{1}=s_{3} s_{1} s_{2} s_{4}$ and $w_{2}=s_{3} s_{1} s_{2} s_{3}$, and these are the row-reading reduced expressions introduced in Section 7

6.3. Excited Young diagrams for shifted cases. The notion of excitation of shifted Young diagrams is defined in the same way as in the case of ordinary Young diagrams if we introduce the idea of elementary excitation.

Let $\mu$ be a strict partition, and let $C$ be a subset of $D_{\mu}^{\prime}$. If a box $x \in C$ satisfies either of the following conditions:

(1) $x=(i, j), i<j$ and $x+(1,0), x+(0,1), x+(1,1) \in D_{\mu}^{\prime} \backslash C$,

(2) $x=(i, i)$ and $x+(0,1), x+(1,1) \in D_{\mu}^{\prime} \backslash C$,

then we set $C^{\prime}=C \cup\{x+(1,1)\} \backslash\{x\}$ and call this procedure $C \rightarrow C^{\prime}$ an elementary excitation of type $I$ occurring at $x \in C$. In general if a subset $S$ of $D_{\mu}^{\prime}$ is obtained from $C$ by applying elementary excitations of type $I$ successively, then we say that $S$ is an excited state of $C$. Suppose we are given a strict partition $\lambda$ such that $\lambda \leq \mu$. Let us denote by $\mathcal{E}_{\mu}^{I}(\lambda)$ the set of all excited states of $D_{\lambda}^{\prime}$. 
We also define the set $\mathcal{E}_{\mu}^{I I}(\lambda)$ consisting of the elements obtained from $C$ by a successive application of elementary excitations of "type $I I$ " defined below. Let $C$ be a subset of $D_{\mu}^{\prime}$, and suppose we take a box $x \in C$ satisfying either of the following conditions:

(1) $x=(i, j), i<j$ and $x+(1,0), x+(0,1), x+(1,1) \in D_{\mu}^{\prime} \backslash C$,

(2) $x=(i, i)$ and $x+(0,1), x+(1,1), x+(1,2), x+(2,2) \in D_{\mu}^{\prime} \backslash C$.

In the case of (1) we set $C^{\prime}=C \cup\{x+(1,1)\} \backslash\{x\}$, and in the case of (2) $C^{\prime}=C \cup\{x+(2,2)\} \backslash\{x\}$. We call the procedure $C \rightarrow C^{\prime}$ an elementary excitation of type $I I$ occurring at $x \in C$. Clearly we have $\mathcal{E}_{\mu}^{I I}(\lambda) \subset \mathcal{E}_{\mu}^{I}(\lambda)$.

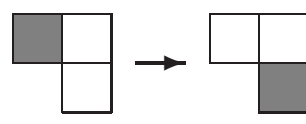

Type I

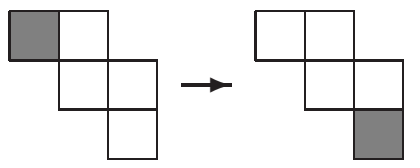

Type II

Example 6.3. Let $\lambda=(3,1), \mu=\rho_{4}$. The set $\mathcal{E}_{\mu}^{I}(\lambda)$ consists of the following ten elements:
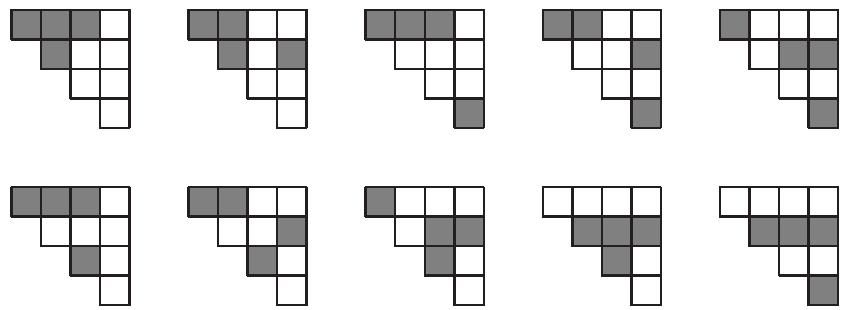

The five members in the first row form the subset $\mathcal{E}_{\mu}^{I I}(\lambda)$. The corresponding elements in $W^{P}$ are as follows: for type $D_{5}: v=5 \overline{4} \overline{3} \overline{2} \overline{1}, w=135 \overline{4} \overline{2}$, for type $C_{4}$ or $B_{4}: v=\overline{4} \overline{3} \overline{2} \overline{1}, w=13 \overline{4} \overline{2}$.

Theorem 6.4. Let $w \leq v \in W^{P}$ and $\lambda \leq \mu$ be the corresponding strict partitions. We have the following formulas:

(1) Type $C_{n}:\left.\left[X_{w}\right]\right|_{v}=\sum_{C \in \mathcal{E}_{\mu}^{I}(\lambda)} \prod_{(i, j) \in C}\left(\varepsilon_{v(n+j)}-\varepsilon_{v(n-i+1)}\right)$,

(2) Type $B_{n}:\left.\left[X_{w}\right]\right|_{v}=\sum_{C \in \mathcal{E}_{\mu}^{I}(\lambda)} \prod_{(i, j) \in C} 2^{-\delta_{i j}}\left(\varepsilon_{v(n+j)}-\varepsilon_{v(n-i+1)}\right)$,

(3) Type $D_{n+1}:\left.\left[X_{w}\right]\right|_{v}=\sum_{C \in \mathcal{E}_{\mu}^{I I}(\lambda)} \prod_{(i, j) \in C}\left(\varepsilon_{v(n+j+2)}-\varepsilon_{v(n-i+2)}\right)$.

Example 6.5. Let $n=4$ and $\mu=\rho_{4}$. We arrange positive roots in $D_{\mu}^{\prime}$ as follows:

\begin{tabular}{|c|l|l|l|}
\hline$\varepsilon_{1}+\varepsilon_{2}$ & $\varepsilon_{1}+\varepsilon_{3}$ & $\varepsilon_{1}+\varepsilon_{4}$ & $\varepsilon_{1}-\varepsilon_{5}$ \\
\hline \multirow{2}{*}{} & $\varepsilon_{2}+\varepsilon_{3}$ & $\varepsilon_{2}+\varepsilon_{4}$ & $\varepsilon_{2}-\varepsilon_{5}$ \\
\hline \multicolumn{1}{|c}{$\varepsilon_{3}+\varepsilon_{4}$} & $\varepsilon_{3}-\varepsilon_{5}$ \\
\cline { 2 - 3 } Type $D_{5}$ & & $\varepsilon_{4}-\varepsilon_{5}$ \\
\cline { 2 - 3 }
\end{tabular}

\begin{tabular}{|l|l|l|l|}
\hline $2 \varepsilon_{1}$ & $\varepsilon_{1}+\varepsilon_{2}$ & $\varepsilon_{1}+\varepsilon_{3}$ & $\varepsilon_{1}+\varepsilon_{4}$ \\
\hline \multirow{4}{*}{$2 \varepsilon_{2}$} & $\varepsilon_{2}+\varepsilon_{3}$ & $\varepsilon_{2}+\varepsilon_{4}$ \\
\hline \multirow{3}{*}{ Type $C_{4}$} & $2 \varepsilon_{3}$ & $\varepsilon_{3}+\varepsilon_{4}$ \\
\cline { 2 - 3 } & $2 \varepsilon_{4}$ \\
\hline
\end{tabular}


Let $\lambda=(2)$. Then the set $\mathcal{E}_{\mu}^{I}(\lambda)$ consists of the following six elements:
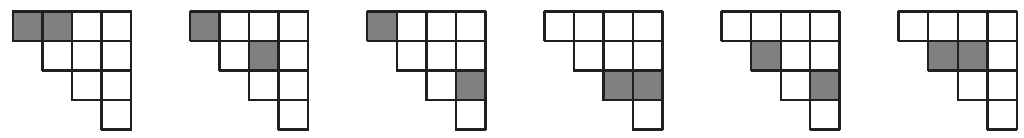

The first four elements form the subset $\mathcal{E}_{\mu}^{I I}(\lambda)$. In the type $D_{5}$ case we have $\left.\left[X_{w}\right]\right|_{v}=\left(\varepsilon_{1}+\varepsilon_{2}\right)\left(\varepsilon_{1}+\varepsilon_{3}\right)+\left(\varepsilon_{1}+\varepsilon_{2}\right)\left(\varepsilon_{2}+\varepsilon_{4}\right)+\left(\varepsilon_{1}+\varepsilon_{2}\right)\left(\varepsilon_{3}-\varepsilon_{5}\right)+\left(\varepsilon_{3}+\varepsilon_{4}\right)\left(\varepsilon_{3}-\varepsilon_{5}\right)$, in the type $C_{4}$ case we have

$$
\begin{aligned}
{\left.\left[X_{w}\right]\right|_{v}=} & 2 \varepsilon_{1}\left(\varepsilon_{1}+\varepsilon_{2}\right)+2 \varepsilon_{1}\left(\varepsilon_{2}+\varepsilon_{3}\right)+2 \varepsilon_{1}\left(\varepsilon_{3}+\varepsilon_{4}\right) \\
& +2 \varepsilon_{3}\left(\varepsilon_{3}+\varepsilon_{4}\right)+2 \varepsilon_{2}\left(\varepsilon_{3}+\varepsilon_{4}\right)+2 \varepsilon_{2}\left(\varepsilon_{2}+\varepsilon_{3}\right) .
\end{aligned}
$$

\section{Proof of Theorem 6.4}

This section is devoted to the proof of Theorem 6.4. Our strategy is the same as in Section 4 .

We shall give the notion of a row-reading expression for each element of $W^{P}$. First we fill in the diagram $D_{\rho_{n}}^{\prime}$ the simple reflections.

Types $B_{n}, C_{n}$ : We define a map $s: D_{\rho_{n}}^{\prime} \rightarrow\left\{s_{1}, \ldots, s_{n}\right\}$ by

where $1 \leq i<j \leq n$.

$$
s(i, j)=\left\{\begin{array}{l}
s_{n} \quad(i=j), \\
s_{n+i-j} \quad(i<j),
\end{array}\right.
$$

Type $D_{n+1}$ : We define a map $s: D_{\rho_{n}}^{\prime} \rightarrow\left\{s_{1}, \ldots, s_{n+1}\right\}$ by

$$
s(i, j)=\left\{\begin{array}{l}
s_{n+1} \quad(i=j, i: \text { odd }), \\
s_{n} \quad(i=j, i: \text { even }), \\
s_{n+i-j} \quad(i<j),
\end{array}\right.
$$

where $1 \leq i<j \leq n$.

Example 7.1. Let $n=4$. The map $s$ is illustrated as follows:

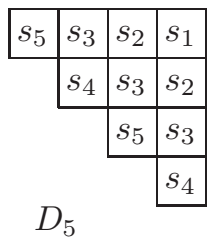

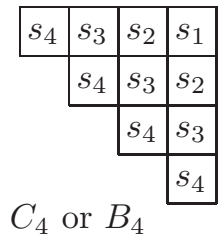

Let $\lambda$ be the strict partition associated to $w \in W^{P}$. We define the row-reading map $\varphi: D_{\lambda}^{\prime} \rightarrow\{1,2, \ldots, k\}$ as in Section 4. We define the word

$$
s_{i_{1}} \cdots s_{i_{k}}
$$

where $s_{i_{j}}=s\left(\varphi^{-1}(j)\right)$. Then we have $w=s_{i_{1}} \cdots s_{i_{k}}$, which is a reduced expression for $w \in W^{P}$. For each subset $C$ of $D_{\mu}^{\prime}$, we define an element $w_{C} \in W$ in the same way in Section 4 . Let $w \leq v \in W^{P}$. The set

$$
\left\{C \subset D_{\mu}^{\prime} \mid \sharp C=\ell(w), w_{C}=w\right\}
$$

is denoted by $\mathcal{R}_{v}^{I}(w)$ (resp. $\left.\mathcal{R}_{v}^{I I}(w)\right)$ for types $C_{n}, B_{n}$ (type $D_{n+1}$ ). 
The key result to prove Theorem 6.4 is the following.

Proposition 7.2. Let $w \leq v \in W^{P}$ and $\lambda \leq \mu$ be the associated strict partitions. Then we have $\mathcal{E}_{\mu}^{*}(\lambda)=\mathcal{R}_{v}^{*}(w)$ for $*=I, I I$.

Lemma 7.3. Let $C, C^{\prime}$ be subsets of a shifted Young diagram $D_{\mu}^{\prime}$ such that $C^{\prime}$ is obtained from $C$ by an elementary excitation of type $*=I$, II. If $C$ belongs to $\mathcal{R}_{v}^{*}(w)$, then we also have $C^{\prime} \in \mathcal{R}_{v}^{*}(w)$.

Proof. Suppose $C^{\prime}$ is obtained from $C$ by an elementary excitation of Type $I I$ occurring at $(i, i)$. Any simple reflection contained in the region $R_{b} \cap C$ are one of $s_{1}, \ldots, s_{n-2}$, each of which commutes with $s_{n+1}$ and $s_{n}$.

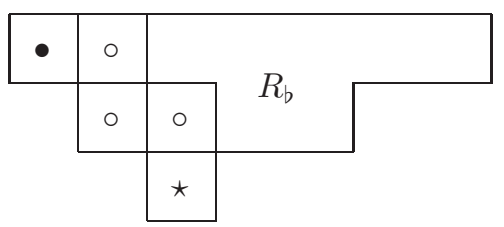

The positions indicated by o are vacant by the definition of elementary excitation. Therefore the word $w_{C^{\prime}}$ can be obtained from $w_{C}$ by using only commuting relations. Hence we have $C^{\prime} \in \mathcal{R}_{v}(w)$. The arguments for the other cases are simpler than this, and we leave them to the reader.

Lemma 7.4. Let $C \in \mathcal{R}_{v}^{*}(w)$ be such that $D_{\lambda}^{\prime} \neq C$. Then there exists $C^{\prime} \in \mathcal{R}_{v}^{*}(w)$ such that $C$ is obtained from $C^{\prime}$ by excitation of type $*$.

Proof. Similar to the proof of Lemma 4.4 .

Proof of Proposition 7.2. This is immediate from Lemmas 7.3, 7.4

Now we calculate the $\beta$-sequence in Proposition 2.3 .

Lemma 7.5. Let $\mu \in \mathcal{S P}_{\rho_{n}}$ and $v$ be the associated element of $W^{P}$. Denote by $\varphi: \mu \longrightarrow\{1,2, \ldots, k\}$ the row-reading map of $\mu$. For $(i, j) \in D_{\mu}^{\prime}$ set $\beta_{i, j}=\beta_{\varphi(i, j)}$, where $\beta_{t}(1 \leq t \leq l)$ are defined in (2.3). We have the following formulas:

(1) Type $C_{n}: \beta_{i, j}=\varepsilon_{v(n+j)}-\varepsilon_{v(n-i+1)}$,

(2) Type $B_{n}: \beta_{i, j}=2^{-\delta_{i j}}\left(\varepsilon_{v(n+j)}-\varepsilon_{v(n-i+1)}\right)$,

(3) Type $D_{n+1}: \beta_{i, j}=\varepsilon_{v(n+2+j)}-\varepsilon_{v(n-i+2)}$.

Proof. Similar to the proof of Lemmas 4.5 and 4.6 .

Proof of Theorem 6.4. The same as the proof of Theorem 3.2 .

\section{Factorial $Q$ - and $P$-Functions}

In this section, we first define the factorial $Q$ - and $P$-functions and present some fundamental properties. Then we use them to express the restriction of the equivariant Schubert classes for the Lagrangian and orthogonal Grassmannians. As an application we give the equivariant Giambelli formula. 
8.1. Factorial $Q$ - and $P$-functions. We first recall the definition of factorial $Q$ and $P$-functions due to Ivanov [11]. Let $\mathcal{S P}(n)$ denote the set of strict partitions $\lambda=\left(\lambda_{1}>\cdots>\lambda_{r}>0\right)$ with $r \leq n$.

Definition 8.1. Let $\lambda \in \mathcal{S P}(n)$. Put

$$
P_{\lambda}^{(n)}(x \mid a)=\frac{1}{(n-r) !} \sum_{w \in S_{n}} w\left(\left(x_{1} \mid a\right)^{\lambda_{1}} \cdots\left(x_{r} \mid a\right)^{\lambda_{r}} \prod_{1 \leq i \leq r, i<j \leq n} \frac{x_{i}+x_{j}}{x_{i}-x_{j}}\right)
$$

where $w \in S_{n}$ acts as a permutation of variables $x_{1}, \ldots, x_{n}$. We also put $Q_{\lambda}^{(n)}(x \mid a)=$ $2^{r} P_{\lambda}^{(n)}(x \mid a)$.

The rational expression on the right hand side of (8.1) is actually a polynomial in $x_{1}, \ldots, x_{n}$ and $a_{1}, a_{2}, \ldots, a_{\lambda_{1}}$, homogeneous of degree $|\lambda|$. In particular we have

$$
P_{1}^{(n)}(x \mid a)= \begin{cases}x_{1}+\cdots+x_{n} & \text { if } n \text { is even, } \\ x_{1}+\cdots+x_{n}-a_{1} & \text { if } n \text { is odd. }\end{cases}
$$

In [11, the first parameter $a_{1}$ is assumed to be zero in most of the argument. However, we need $a_{1}$ for later use. In order to generalize Ivanov's results to the case of non-zero $a_{1}$ the following is fundamental (cf. [11, Proposition 2.6).

Proposition 8.2 (Stability mod 2). For any $\lambda \in \mathcal{S P}(n)$, we have

$$
P_{\lambda}^{(n+2)}\left(x_{1}, \ldots, x_{n}, 0,0 \mid a\right)=P_{\lambda}^{(n)}\left(x_{1}, \ldots, x_{n} \mid a\right) .
$$

Proof. We set

$$
F_{n}=\left(x_{1} \mid a\right)^{\lambda_{1}} \cdots\left(x_{r} \mid a\right)^{\lambda_{r}} \prod_{1 \leq i \leq r, i<j \leq n} \frac{x_{i}+x_{j}}{x_{i}-x_{j}}
$$

Let $w \in S_{n+2}$, and consider the term $w\left(F_{n+2}\right)$. If $w(i) \neq n+1, n+2$ for all $i=1, \ldots, r$, then the rational function $w\left(F_{n+2}\right)$ has no pole along the hyperplane $x_{n+1}-x_{n+2}=0$. So we can substitute $x_{n+1}=x_{n+2}=0$ to such a rational function. As the result of the substitution we have $w^{\prime}\left(F_{n}\right)$ for some $w^{\prime} \in S_{n}$. In fact, $w^{\prime}$ is obtained by eliminating $n+1, n+2$ from the sequence $w(1), \ldots, w(n+2)$. By this correspondence, there are $(n-r+2)(n-r+1)$ elements of $S_{n+2}$ giving the same $w^{\prime} \in S_{n}$. Thus our task is to show that all the remaining terms cancel out.

Let $i=w^{-1}(n+1), j=w^{-1}(n+2)$. Suppose $i \leq r$ or $j \leq r$. We set $w^{\prime}=$ $w \cdot(i, j)$. Then both $w\left(F_{n+2}\right)$ and $w^{\prime}\left(F_{n+2}\right)$ have a simple pole along $x_{n+1}-x_{n+2}=$ 0 ; however, the sum $w\left(F_{n+2}\right)+w^{\prime}\left(F_{n+2}\right)$ has no pole along $x_{n+1}-x_{n+2}=0$. Substituting $x_{n+1}=x_{n+2}=0$ to the sum, we obtain zero.

8.2. Vanishing property. Here we present a vanishing property of $P_{\lambda}^{(n)}(x \mid a)$. For a strict partition $\lambda=\left(\lambda_{1}>\cdots>\lambda_{r}>0\right)$ with $r \leq n$ define an $n$-tuple

$$
a_{\lambda}=\left\{\begin{array}{lll}
\left(a_{\lambda_{1}+1}, \ldots, a_{\lambda_{r}+1}, 0, \ldots, 0\right) & \text { if } n-r \text { is even } \\
\left(a_{\lambda_{1}+1}, \ldots, a_{\lambda_{r}+1}, a_{1}, 0, \ldots, 0\right) & \text { if } n-r \text { is odd }
\end{array}\right.
$$

Proposition 8.3. We have $P_{\lambda}^{(n)}\left(a_{\mu} \mid a\right)=0$ unless $\mu \geq \lambda$.

Proof. By Proposition 8.2 we may assume $a_{\mu}$ has no zero entries. Then the proposition follows from the definition. 
8.3. Pieri's rule and Pfaffian formulas. Here we collect some basic facts on the factorial $P$-Schur functions, which we shall make use of in the next subsection. The proof for the $a_{1}=0$ case is given in [11]. The same proof works for the general $a_{1}$ case using the vanishing property.

Proposition 8.4 ([1] $)$. For a strict partition $\lambda \in \mathcal{S P}(n)$ we have

$$
\left(P_{1}^{(n)}(x \mid a)-P_{1}^{(n)}\left(a_{\lambda} \mid a\right)\right) P_{\lambda}^{(n)}(x \mid a)=\sum_{\lambda \rightarrow \nu} P_{\nu}^{(n)}(x \mid a)
$$

where the sum is over $\nu \in \mathcal{S P}(n)$ such that $\nu \geq \lambda$ and $|\nu|=|\lambda|+1$.

For any $\lambda=\left(\lambda_{1}>\cdots>\lambda_{r}>0\right) \in \mathcal{S P}(n)$, set $r_{0}(\lambda)=r$ if $r$ is even and $r_{0}(\lambda)=r+1$ if $r$ is odd, and then we put $\lambda_{r+1}=0$.

Lemma 8.5 ([1]). For a strict partition $\lambda \in \mathcal{S P}(n)$ we have

$$
P_{\lambda}^{(n)}(x \mid a)=\operatorname{Pf}\left(P_{\lambda_{i}, \lambda_{j}}^{(n)}(x \mid a)\right)_{1 \leq i<j \leq r_{0}(\lambda)} .
$$

8.4. Closed formula for $\left.\left[X_{w}\right]\right|_{v}$ and the equivariant Giambelli formula. For each $v \in W^{P}$, we shall define an $n$-tuple $x_{v}$ in the following way:

Types $C_{n}$ and $B_{n}$ : Let $v \in W^{P}$, and

$$
\{i \mid 1 \leq i \leq n, v(i)>n\}=\left\{\bar{j}_{1}, \ldots, \bar{j}_{k}\right\},
$$

where $1 \leq j_{1}<\cdots<j_{k} \leq n$. Then we put

$$
x_{v}=\left(\varepsilon_{j_{1}}, \ldots, \varepsilon_{j_{k}}, 0, \ldots, 0\right) .
$$

Type $D_{n+1}$ : Let $v \in W^{P}$, and

$$
\{i \mid 1 \leq i \leq n+1, v(i)>n+1\}=\left\{\bar{j}_{1}, \ldots, \bar{j}_{k}\right\}
$$

where $1 \leq j_{1}<\cdots<j_{k} \leq n+1$. Note that $k$ is even. If $n$ is even we put

$$
x_{v}=\left(\varepsilon_{j_{1}}, \ldots, \varepsilon_{j_{k}}, 0, \ldots, 0\right) \text {. }
$$

If $n$ is odd, let $r=r(\lambda)$, where $\lambda$ is the strict partition corresponding to $v$, and put

$$
x_{v}= \begin{cases}\left(\varepsilon_{j_{1}}, \ldots, \varepsilon_{j_{r}}, 0, \ldots, 0\right) & \text { if } n-r \text { is odd } \\ \left(\varepsilon_{j_{1}}, \ldots, \varepsilon_{j_{r}},-\varepsilon_{n+1}, 0, \ldots, 0\right) & \text { if } n-r \text { is even. }\end{cases}
$$

Lemma 8.6. Let $v \in W^{P}$. We have

(1) Type $C_{n}:\left.\left[X_{s_{n}}\right]\right|_{v}=Q_{1}^{(n)}\left(x_{v} \mid 0, \varepsilon_{n}, \ldots, \varepsilon_{2}\right)$,

(2) Type $B_{n}:\left.\left[X_{s_{n}}\right]\right|_{v}=P_{1}^{(n)}\left(x_{v} \mid 0, \varepsilon_{n}, \ldots, \varepsilon_{2}\right)$,

(3) Type $D_{n+1}:\left.\left[X_{s_{n+1}}\right]\right|_{v}=P_{1}^{(n)}\left(x_{v} \mid(-1)^{n} \varepsilon_{n+1}, \varepsilon_{n}, \ldots, \varepsilon_{2}\right)$.

Proof. Consider Type $D_{n+1}$. By Proposition 2.1 (4), and (6.1), we have

$$
\left.\left[X_{s_{n+1}}\right]\right|_{v}=\varpi_{n+1}-v\left(\varpi_{n+1}\right)=\sum_{1 \leq i \leq n+1, v(i)>n+1} \varepsilon_{i} .
$$

Now recall the form of $P_{1}(x \mid a)$ given by 8.2 . Then it is easy to check our formula. Types $C_{n}$ and $B_{n}$ are similar and much simpler.

Now we can state the main result of this section.

Theorem 8.7. Let $w \leq v \in W^{P}$ and $\lambda \leq \mu$ be the corresponding strict partitions. We have the following formulas:

(1) Type $C_{n}([10]):\left.\left[X_{w}\right]\right|_{v}=Q_{\lambda}^{(n)}\left(x_{v} \mid 0, \varepsilon_{n}, \ldots, \varepsilon_{2}\right)$, 
(2) Type $B_{n}:\left.\left[X_{w}\right]\right|_{v}=P_{\lambda}^{(n)}\left(x_{v} \mid 0, \varepsilon_{n}, \ldots, \varepsilon_{2}\right)$,

(3) Type $D_{n+1}:\left.\left[X_{w}\right]\right|_{v}=P_{\lambda}^{(n)}\left(x_{v} \mid(-1)^{n} \varepsilon_{n+1}, \varepsilon_{n}, \ldots, \varepsilon_{2}\right)$.

Proof. The result for Type $C_{n}$ has been proved in [10. We consider Type $D_{n+1}$. Assume $n$ is even for simplicity. The odd case is left for the reader. Fix $\mu \in$ $\mathcal{S} \mathcal{P}_{\rho_{n}}$. Consider the following system of equations for the functions $F_{\lambda}\left(\varepsilon_{1}, \ldots, \varepsilon_{n+1}\right)$ $\left(\lambda \in \mathcal{S P}_{\mu}\right):$

$$
\left(\sum_{i=1}^{r_{0}(\mu)} \varepsilon_{n-\mu_{i}+1}-\sum_{i=1}^{r_{0}(\lambda)} \varepsilon_{n-\lambda_{i}+1}\right) F_{\lambda}(\varepsilon)=\sum_{\nu} F_{\nu}(\varepsilon) \quad \text { for all } \lambda \in \mathcal{S P} \mathcal{P}_{\mu} \backslash\{\mu\}
$$

where the summation on the right hand side runs through $\nu \in \mathcal{S P}_{\mu}$ such that $\nu \geq \lambda$ and $|\nu|=|\lambda|+1$. By equation (5.3) together with the initial condition $F_{\phi}\left(\varepsilon_{1}, \ldots, \varepsilon_{n}\right)=1$, the set of functions $F_{\lambda}(\varepsilon)\left(\lambda \in \mathcal{S P}_{\mu}\right)$ is characterized.

By equation (2.2) for type $D_{n+1},\left.\left[X_{w}\right]\right|_{v}=\xi^{w}(v)$ (cf. Proposition 2.2) satisfies equation (8.4) as well as the initial condition (cf. Proposition 2.1 (1)). Note that the coefficients $\left\langle w\left(\varpi_{n+1}\right), \beta^{\vee}\right\rangle$, the Chevalley multiplicity, is equal to one on the right hand side of equation (2.2), i.e., $G / P$ in this case is 'minuscule'. On the other hand, the functions on the right hand side of the formula in the theorem also satisfy (8.4). This fact is a consequence of Proposition 8.4 and the vanishing property (Proposition 8.3) of $P_{\lambda}^{(n)}(x \mid a)$. The initial condition is also satisfied. Therefore we have the theorem. The type $B_{n}$ case is quite similar to the case of type $D_{n+1}$.

The following result is a direct consequence of Theorem 8.7 and Lemma 8.5.

Corollary 8.8 (Equivariant Giambelli). Let $G / P$ be of type $C_{n}, B_{n}, D_{n+1}$. For any $\lambda \in \mathcal{S P}_{\rho_{n}}$, we have

$$
\left[X_{\lambda}\right]=\operatorname{Pf}\left(\left[X_{\lambda_{i}, \lambda_{j}}\right]\right)_{1 \leq i<j \leq r_{0}(\lambda)},
$$

where we denote by $X_{\lambda}$ the Schubert variety corresponding to $\lambda$.

Proof. The proof is the same as that given in 10 .

\section{Multiplicity of a Singular point in a Schubert Variety}

Another application is to the multiplicity of a singular point in a Schubert variety. We denote the multiplicity of the variety $X_{w}$ at $e_{v}$ by $m_{v}\left(X_{w}\right)$. We will explain the relationship between $\left.\left[X_{w}\right]\right|_{v}$ and $m_{v}\left(X_{w}\right)$. Then we discuss some implications of our result on $m_{v}\left(X_{w}\right)$.

Let $R_{\text {uni }}(P)$ be the unipotent radical of $P$, and let $R_{P}^{+}$be the subset of $R^{+}$defined by $R_{P}^{+}=\left\{\beta \in R \mid U_{\beta} \subset R_{\text {uni }}(P)\right\}$, where $U_{\beta}$ is the root subgroup associated to $\beta$. For a given $v \in W^{P}$, let $U_{v}^{-}$be the subgroup of $G$ generated by the root subgroups $U_{-\beta}, \beta \in v\left(R^{+} \backslash R_{P}^{+}\right)$. Under the map $G \rightarrow G / P, U_{v}^{-}$is mapped isomorphically onto its image $\mathcal{U}_{v}:=U_{v}^{-} e_{v}$, which is a canonical $T$-stable affine neighborhood of $e_{v}$ with a coordinate system $\left\{x_{-\beta} \mid \beta \in v\left(R^{+} \backslash R_{P}^{+}\right)\right\}$.

Let $G / P$ be either of types $A_{n-1}, C_{n}$, or $D_{n}$. For $w, v \in W^{P}, v \geq w$, let us denote $Y_{w, v}=X_{w} \cap \mathcal{U}_{v}$. It is known that in these cases the defining ideal of the affine variety $Y_{w, v}$ is homogeneous in our coordinate system, i.e., $Y_{w, v}$ is a cone in $\mathcal{U}_{v}$. Actually we have a one parameter subgroup $\phi_{v} \in \operatorname{Hom}\left(\mathbb{C}^{\times}, T\right)$ where $\phi_{v}(t)\left(t \in \mathbb{C}^{\times}\right)$acts on $\mathcal{U}_{v}$ by dilation. Then there exists $h_{v} \in \operatorname{Lie}(T)$ such that $h_{v}(\beta)=-1$ for all $\beta \in v\left(R^{+} \backslash R_{P}^{+}\right)$. For example, if $v \in W\left(C_{n}\right)^{P_{n}}$, then $h_{v}$ is given by $h_{v}\left(\varepsilon_{i}\right)=\frac{1}{2}$ if $v(i)>n$ and $-\frac{1}{2}$ if $v(i) \leq n$, for $1 \leq i \leq n$. Note that each $\left.\left[X_{w}\right]\right|_{v} \in S$, being a 
polynomial function on $\operatorname{Lie}(T)$, can be evaluated at $h_{v}$. For background of the next proposition we refer to [27.

Proposition 9.1. Let $G / P$ be either of types $A_{n-1}, C_{n}, D_{n}$ and $w \leq v \in W^{P}$. The value of $\left.\left[X_{w}\right]\right|_{v}$ evaluated at $h_{v}$ gives the multiplicity of the variety $X_{w}$ at $e_{v}$.

Proof. Consider the neighborhood $\mathcal{U}_{v}$ of $e_{v}$. The multiplicity $m_{v}\left(X_{w}\right)$ is determined by the Poincaré series of the coordinate ring $\mathbb{C}\left[Y_{w, v}\right]$, which is given by restricting the formal character $\operatorname{ch} \mathbb{C}\left[Y_{w, v}\right]$ as a $T$-module to the one parameter subgroup $\phi_{v}$ associated with $h_{v}$. Then $\left.\left[X_{w}\right]\right|_{v}$ evaluated at $h_{v}$ is nothing but the classical multiplicity in the sense of Hilbert-Samuel.

Remark. There are recurrence relations for $m_{v}\left(X_{w}\right)$ given by Lakshmibai and Weyman [23, which can be obtained by specializing equations (5.3), (8.4) at $h_{v}$. So we have another proof of the above proposition.

Corollary 9.2 ([19, [12, [17]). Let $G / P=\mathcal{G}_{d, n}, w \leq v \in W^{P_{d}}$, and $\lambda \leq \mu \in \mathcal{P}_{d, n}$ be the corresponding partitions. Then we have

$$
m_{v}\left(X_{w}\right)=\sharp \mathcal{E}_{\mu}(\lambda) .
$$

Proof. This is immediate from Proposition 9.1 and Theorem 3.2 since each summand on the right hand side of the formula becomes one, when specialized at $h_{v}$.

Remark 2. This formula can be obtained as a direct consequence of the result in [19, 12] describing the Gröbner basis of the defining ideal of $Y_{w, v}$, together with a combinatorial argument in [17].

Corollary 9.3. Let $G / P$ be the Grassmannian of types $C_{n}$ or $D_{n+1}$. Let $w \leq v \in$ $W^{P}$ and $\lambda \leq \mu \in \mathcal{S P}_{\rho_{n}}$ be the corresponding strict partitions. Then we have

(1) $\left([8,[18])\right.$ Type $C_{n}$ :

$$
m_{v}\left(X_{w}\right)=\sharp \mathcal{E}_{\mu}^{I}(\lambda),
$$

(2) Type $D_{n+1}$ :

$$
m_{v}\left(X_{w}\right)=\sharp \mathcal{E}_{\mu}^{I I}(\lambda) .
$$

Proof. The same as Corollary 9.2 .

Example 9.4 (cf. Example 6.3). Let $v=5 \overline{4} \overline{3} \overline{2} \overline{1}, w=135 \overline{4} \overline{2}$ (type $D_{5}$ ), and $v=\overline{4} \overline{3} \overline{2} \overline{1}, w=13 \overline{4} \overline{2}$ (types $B_{4}$ or $C_{4}$ ). Then we have $m_{v}\left(X_{w}\right)=10$ for $C_{4}$ and $m_{w}\left(X_{v}\right)=5$ for $B_{4}, D_{5}$.

Remark 3. We should make a remark on (9.2) similar to Remark 2. Ghorpade and Raghavan [8] have given a detailed description of the Gröbner basis of the defining ideal of $Y_{w, v}$ for the Lagrangian Grassmannian. Then (9.2) can be derived from a result in [18. As for Type $D_{n+1}$, formula (9.3) seems to be new (see the note added in Section 1).

We close this section by pointing out the following fact.

Proposition 9.5. With notation as in Corollary 9.3, we have

$$
m_{v}\left(X_{w}\right)=\operatorname{Pf}\left(m_{v}\left(X_{\lambda_{i}, \lambda_{j}}\right)\right)_{1 \leq i<j \leq r_{0}(\lambda)} .
$$

Proof. This is immediate from Corollary 8.8 and Proposition 9.1 


\section{LATTICE PATHS METHOD}

As a supplementary discussion, we will show a direct combinatorial route from the combinatorial formula (Theorem 6.4) to the equivariant Giambelli formula (Corollary 8.8). Our argument relies on the "lattice path method" due to Stembridge. In order to deal with the case of type $D_{n+1}$ also, we slightly modify the results in 29$]$.

10.1. Perfect matchings. Let $r$ be a positive even integer and consider a set of $r$ elements $U_{r}=\left\{u_{1}, \ldots, u_{r}\right\}$. A perfect matching on $U_{r}$ is a partition of the set $U_{r}$ with each part consisting of two element subsets. We denote a perfect matching $\sigma$ on $U_{r}$ in such a way that $\sigma=\left\{\left(u_{i_{k}}, u_{j_{k}}\right)\right\}_{k=1, \ldots, r / 2}$, where $i_{k}<j_{k}(1 \leq k \leq r / 2), i_{1}<$ $\cdots<i_{r / 2}$, and put $I_{\sigma}=\left\{i_{1}, \ldots, i_{r / 2}\right\}$. The signature $\operatorname{sgn}(\sigma)$ of $\sigma$ is defined as $(-1)^{c(\sigma)}$, where $c(\sigma)=\#\left\{(i, j, k, l) \mid 1 \leq i<j<k<l \leq r,\left(u_{i}, u_{k}\right),\left(u_{j}, u_{l}\right) \in \sigma\right\}$ is the number of crossings in $\sigma$ (which depends on the numbering of elements of $U_{r}$, but we fix it once and for all). Denote by $\mathcal{M}_{r}$ the set of all perfect matchings on the set $U_{r}$ and let $\sigma_{0}$ be the 'identical' perfect matching $\left\{\left(u_{2 i-1}, u_{2 i}\right)\right\}_{i=1}^{r / 2}$.

Lemma 10.1. There is an involution on $\mathcal{M}_{r} \backslash\left\{\sigma_{0}\right\}\left(\sigma \mapsto \sigma^{*}\right)$ such that $\operatorname{sgn}(\sigma)=$ $-\operatorname{sgn}\left(\sigma^{*}\right)$ and $I_{\sigma}=I_{\sigma^{*}}$.

Proof. Take the smallest number $k$ such that $\left(u_{i}, u_{2 k-1}\right),\left(u_{j}, u_{2 k}\right) \in \sigma$. Define a perfect matching $\sigma^{\prime}$ by replacing $\left(u_{i}, u_{2 k-1}\right),\left(u_{j}, u_{2 k}\right)$ in $\sigma$ into $\left(u_{j}, u_{2 k-1}\right),\left(u_{i}, u_{2 k}\right)$. Then it is easy to see that $\sigma^{*}$ has the desired property.

10.2. Modified version of Stembridge's argument. Let $\mu=\left(\mu_{1}, \ldots, \mu_{l}\right) \in$ $\mathcal{S} \mathcal{P}_{\rho_{n}}$. We define a directed graph as follows. The vertex set is

$$
D_{\mu}^{\prime} \cup\left(D_{\mu}^{\prime}+\boldsymbol{a}\right)
$$

where $\boldsymbol{a}=\left(\frac{1}{2},-\frac{1}{2}\right)$. Direct an edge from $u$ to $v$ if (1) $v-u=\boldsymbol{b}$, with $\boldsymbol{b}=\left(-\frac{1}{2},-\frac{1}{2}\right)$ or (2) $u \in D_{\mu}^{\prime}$ and $v-u=\boldsymbol{a}$. If we take $\mu=\rho_{n}$, the directed graph is the following:

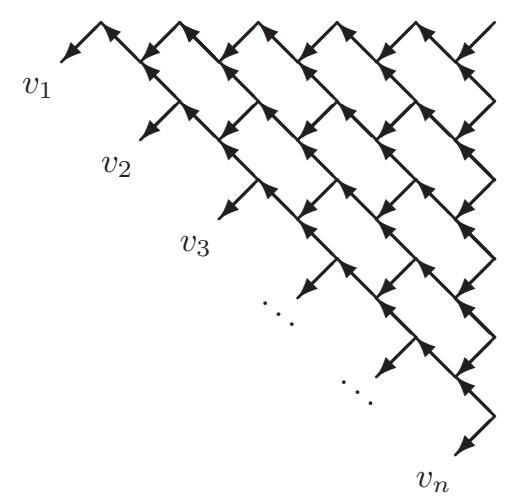

Given a strict partition $\lambda=\left(\lambda_{1}, \ldots, \lambda_{r}\right)$ such that $\lambda \leq \mu$, let $\left(u_{1}, \ldots, u_{r}\right)$ be the $r$-tuple of vertices defined as follows. If $\mu=\rho_{n}$, then we put

$$
u_{i}=\left(n+1-\lambda_{i}, n\right) \text {. }
$$

In general, we put $u_{i}=\left(i+m_{i}, \lambda_{i}+i-1+m_{i}\right)$, where $m_{i}$ is the largest non-negative integer such that $\left(i+m_{i}, \lambda_{i}+i-1+m_{i}\right) \in D_{\mu}^{\prime}$. Define $v_{i}=(i, i)+\boldsymbol{a}$ for $1 \leq i \leq l$ 
and set $J=\left\{v_{1}, \ldots, v_{l}\right\}$, and also

$$
J_{1}=\left\{v_{i} \mid i \text { is odd }\right\}, \quad J_{2}=\left\{v_{i} \mid i \text { is even }\right\} .
$$

For each subset $I$ of $J$ we denote by $\mathcal{P}\left(u_{i}, I\right)$ the set of paths starting from $u_{i}$ to any vertex in $I$. Let $\mathcal{P}_{\mu}^{I}(\lambda)$ denote the set of $r$-tuples of paths $\left(p_{1}, \ldots, p_{r}\right)$ such that $p_{i} \in \mathcal{P}\left(u_{i}, J\right)$ for $1 \leq i \leq r$. Let $\mathcal{P}_{\mu}^{I I}(\lambda)$ denote the subset of $\mathcal{P}_{\mu}^{I}(\lambda)$ such that

$$
p_{i} \in \mathcal{P}\left(u_{i}, J_{i}\right) \quad \text { for all } 1 \leq i \leq r,
$$

where the index $i$ in $J_{i}$ is viewed as modulo 2 . For $*=I$, II, we define $\mathcal{N}_{\mu}^{*}(\lambda)$ to be the set of $r$-tuples $\boldsymbol{p}=\left(p_{1}, \ldots, p_{r}\right)$ of non-intersecting paths in $\mathcal{P}_{\mu}^{*}(\lambda)$.

For $u=(i, j) \in D_{\mu}^{\prime}$, we assign an arbitrary weight $a_{i j}$ (any element in a fixed commutative ring) to the edge $u \rightarrow u+\boldsymbol{a}$. All the other edges are assigned the weight 1 . For the $r$-tuple of paths $\boldsymbol{p}=\left(p_{1}, \ldots, p_{r}\right)$ in $\mathcal{P}_{\mu}^{*}(\lambda)$, let $w\left(p_{i}\right)$ denote the product of all weights which $p_{i}$ go through and put $w(\boldsymbol{p})=w\left(p_{1}\right) \cdots w\left(p_{r}\right)$. Let

$$
\mathcal{W}_{\mu}^{*}(\lambda)=\sum_{\boldsymbol{p} \in \mathcal{N}_{\mu}^{*}(\lambda)} w(\boldsymbol{p})
$$

denote the corresponding generating function.

Proposition 10.2 (29]). Let $\lambda \leq \mu \in \mathcal{S P}_{\rho_{n}}$. We have

$$
\mathcal{W}_{\mu}^{*}(\lambda)=\operatorname{Pf} \mathcal{W}_{\mu}^{*}\left(\lambda_{i}, \lambda_{j}\right)_{1 \leq i<j \leq r_{0}(\lambda)} .
$$

Proof. For the case of $*=I$, we can apply Theorem 3.1 in 29] directly. Here we consider the case of $*=I I$. We may assume that $r=r_{0}(\lambda)$ is even (see the Remark after Theorem 3.1, 29]). Given a perfect matching $\sigma$ of $\left\{u_{1}, \ldots, u_{r}\right\}$, an $r$-tuple of paths $\boldsymbol{p}=\left(p_{1}, \ldots, p_{r}\right) \in \prod_{i=1}^{r} \mathcal{P}\left(u_{i}, J\right)$ is $\sigma$-admissible if for each $\left(u_{i}, u_{j}\right) \in \sigma$ with $i<j, p_{i}$ and $p_{j}$ do not intersect and $p_{i} \in \mathcal{P}\left(u_{i}, J_{1}\right), p_{j} \in \mathcal{P}\left(u_{j}, J_{2}\right)$. Denote the set $\hat{\mathcal{P}}$ of pairs $(\sigma, \boldsymbol{p})$, where $\sigma$ is a perfect matching of $\left\{u_{1}, \ldots, u_{r}\right\}$, and $\boldsymbol{p}=\left(p_{1}, \ldots, p_{r}\right)$ is an $r$-tuple of paths that is $\sigma$-admissible. If we assign the weight $\operatorname{sgn}(\sigma) w(\boldsymbol{p})$ to $(\sigma, \boldsymbol{p}) \in \hat{\mathcal{P}}$, then the Pfaffian on the right hand side of (10.1) is equal to the following generating function:

$$
G(\hat{\mathcal{P}})=\sum_{(\sigma, \boldsymbol{p}) \in \hat{\mathcal{P}}} \operatorname{sgn}(\sigma) w(\boldsymbol{p})
$$

for $\hat{\mathcal{P}}$. Let $\hat{\mathcal{P}}^{\times}$denote the set of $(\sigma, \boldsymbol{p}) \in \hat{\mathcal{P}}$ such that $p_{1}, \ldots, p_{r}$ has at least one intersection, and let $\hat{\mathcal{N}}$ denote the complement of $\hat{\mathcal{P}}^{\times}$in $\hat{\mathcal{P}}$. For any subset $S$ of $\hat{\mathcal{P}}$ let $G(S)$ denote the corresponding generating functions. Then we have

$$
G(\hat{\mathcal{P}})=G(\hat{\mathcal{N}})+G\left(\hat{\mathcal{P}}^{\times}\right) .
$$

We will show that $G\left(\hat{\mathcal{P}}^{\times}\right)=0$. The argument is similar to the one in 30. We construct a sign-reversing involution $(\sigma, \boldsymbol{p}) \mapsto\left(\sigma^{\prime}, \boldsymbol{p}^{\prime}\right)$ on the set $\hat{\mathcal{P}}^{\times}$such that $w(\boldsymbol{p})=w\left(\boldsymbol{p}^{\prime}\right)$. The existence of such an involution implies $G\left(\hat{\mathcal{P}}^{\times}\right)=0$.

Let $v$ be a vertex on a path $p_{i}$. We denote by $p_{i}(\rightarrow v)$ and $p_{i}(v \rightarrow)$ the subpaths of $p_{i}$ from $u_{i}$ to $v$ and $v$ to the end point. We say a vertex $v$ is an intersection point of the $r$-tuple $\boldsymbol{p}=\left(p_{1}, \ldots, p_{r}\right)$ if there are at least one pair of paths that intersect at $v$. Suppose there is an intersecting point of $\boldsymbol{p}=\left(p_{1}, \ldots, p_{r}\right)$. There is an index $i$ that satisfies the following:

- $p_{i}$ intersect $p_{i+1}$ at a vertex $v$,

- there are no intersection points of $\boldsymbol{p}$ on $p_{i}(\rightarrow v)$ and $p_{i+1}(v \rightarrow)$. 
The existence of such an index $i$ can be shown by introducing a total order of the vertices (cf. [29]).

Define paths $p_{i}^{\prime}=p_{i}(\rightarrow v) p_{i+1}(v \rightarrow), p_{i+1}^{\prime}=p_{i+1}(\rightarrow v) p_{i}(v \rightarrow)$, and set $p_{k}^{\prime}=p_{k}$ for $k \neq i, i+1$. Set $\boldsymbol{p}^{\prime}=\left(p_{1}^{\prime}, \ldots, p_{r}^{\prime}\right)$. Also denote by $\sigma^{\prime}$ the perfect matching obtained by interchanging $u_{i}$ and $u_{i+1}$ in $\sigma$. Then we claim that the $r$-tuple $\boldsymbol{p}^{\prime}$ is $\sigma^{\prime}$-admissible. If $\left(u_{k}, u_{l}\right)$ in $\sigma^{\prime}$, then the paths $p_{k}^{\prime}$ and $p_{l}^{\prime}$ do not intersect. For the proof of this fact we refer to the proof of Theorem 3.1 in 29]. Next we have to show that each path $p_{i}^{\prime}(1 \leq i \leq r)$ ends at the right region specified by the perfect matching $\sigma^{\prime}$. Suppose that $p_{i}$ ends at a point in $J_{1}$ and $p_{i+1}$ ends at a point in $J_{2}$. There are indices $k, l$ such that $\left(u_{i}, u_{k}\right),\left(u_{l}, u_{i+1}\right) \in \sigma$ with $i<k, l<i+1$. Note that we have $l<i$ and $i+1<k$ since $\{i, k\}$ and $\{l, i+1\}$ are disjoint. Now by construction, the path $p_{i}^{\prime}$ ends at a point in $J_{2}$ and $p_{i+1}^{\prime}$ ends at a point in $J_{1}$. Since the paths $p_{k}^{\prime}(k \neq i, i+1)$ are not changed we see that all the paths $p_{1}^{\prime}, \ldots, p_{r}^{\prime}$ end at the right regions. Other cases are left for the reader. Thus we have an involution $(\sigma, \boldsymbol{p}) \mapsto\left(\sigma^{\prime}, \boldsymbol{p}^{\prime}\right)$ on $\mathcal{P}^{\times}$with the desired property.

Let $\hat{\mathcal{N}}_{0}$ denote the subset of $\hat{\mathcal{N}}$ consisting of the pairs $\left(\sigma_{0}, \boldsymbol{p}\right)$ such that $\boldsymbol{p} \in \mathcal{N}_{\mu}^{I I}(\lambda)$. Obviously we have $G\left(\hat{\mathcal{N}}_{0}\right)=\mathcal{W}_{\mu}^{I I}(\lambda)$. It remains to show that we can delete all of the terms corresponding to the complementary set $\hat{\mathcal{N}} \backslash \hat{\mathcal{N}}_{0}$. To show this we define a sign-reversing involution on the set $\hat{\mathcal{N}} \backslash \hat{\mathcal{N}}_{0}$ by $(\sigma, \boldsymbol{p}) \mapsto\left(\sigma^{*}, \boldsymbol{p}\right)$, where $\sigma^{*}$ is defined in Lemma 10.1. Note that the condition $I_{\sigma}=I_{\sigma^{*}}$ insures that the $r$-tuple $\boldsymbol{p}$ is $\sigma^{*}$-admissible.

10.3. Bijection between $\mathcal{N}_{\mu}^{*}(\lambda)$ and $\mathcal{E}_{\mu}^{*}(\lambda)$. We will establish a bijection between $\mathcal{N}_{\mu}^{*}(\lambda)$ and $\mathcal{E}_{\mu}^{*}(\lambda)$. The following is an example of a tuple of non-intersecting paths with $\lambda=(4,3,2,1)$ and $\mu=\rho_{6}$ and the corresponding shifted EYD:
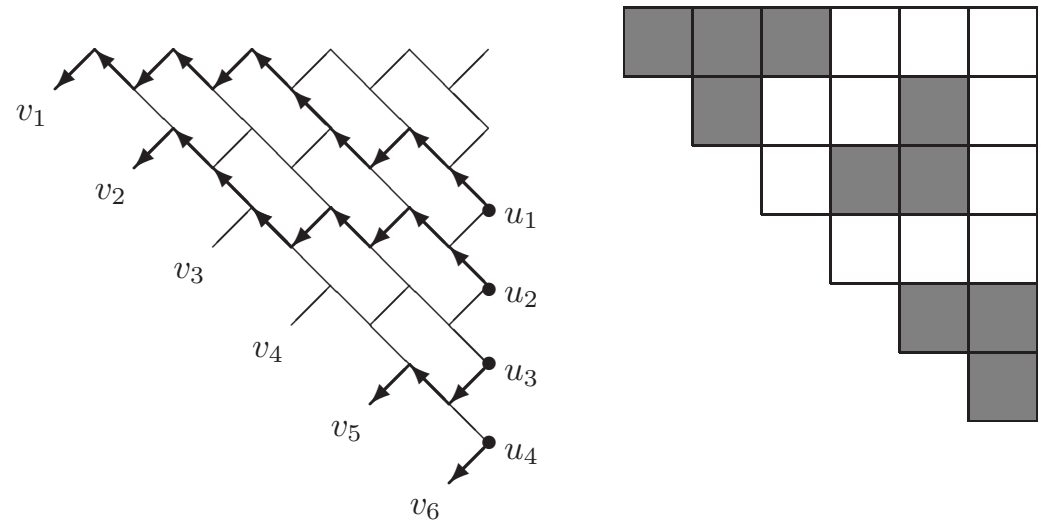

Let $\boldsymbol{p}=\left(p_{1}, \ldots, p_{r}\right) \in \mathcal{N}_{\mu}^{*}(\lambda)$. We define a subset of $D_{\mu}^{\prime}$ by $C(\boldsymbol{p})=\bigcup_{i=1}^{r} C\left(p_{i}\right)$, where $C\left(p_{i}\right)=\left\{v \in D_{\mu}^{\prime} \mid p_{i}\right.$ goes through the edge from $v$ to $\left.v+\boldsymbol{a}\right\}$. First we claim the following.

Proposition 10.3. Let $\boldsymbol{p} \in \mathcal{N}_{\mu}^{*}(\lambda)$. Then $C(\boldsymbol{p})$ is an element of $\mathcal{E}_{\mu}^{*}(\lambda)$.

Proof. We use induction on the energy of $C(\boldsymbol{p})$. Suppose $C(\boldsymbol{p})$ has zero energy.

Then we have $C(\boldsymbol{p})=D_{\lambda}^{\prime}$ and the proposition is true. Let $\boldsymbol{p}^{0}=\left(p_{1}^{0}, \ldots, p_{r}^{0}\right)$ denote the corresponding $r$-tuple with $D_{\lambda}^{\prime}$, the ground state. If $C$ has an energy $>0$, then there is a path $p_{i}$ such that $p_{i} \neq p_{i}^{0}$. We take the smallest index $i$. Let 
$C\left(p_{i}\right)=\left\{w_{1}, \ldots, w_{r}\right\}$, where we arrange the vertices from $w_{1}$ to $w_{r}$ along the path. Take the largest number $k$ such that $w_{k} \notin C\left(p_{i}^{0}\right)$ and put $v=w_{k}$. First consider the case that $v$ is not on the diagonal. Put $x_{1}=v-(1,1), x_{2}=v-(1,0), x_{3}=v-(0,1)$. Then we see that there are no paths that go through either $x_{1}$ or $x_{2}$ from the way of choosing the vertex $v$. Next we claim that $x_{3}$ does not belong to $C(\boldsymbol{p})$. This is clear because the path $p_{i}$ goes straight through the vertex $x_{3}$ to the NW direction. Now we deform the path $p_{i}$ to get $p_{i}^{\prime}$ as in the figure, and set $\boldsymbol{p}^{\prime}=\left(p_{1}^{\prime}, \ldots, p_{r}^{\prime}\right)$ with $p_{j}^{\prime}=p_{j}$ for $j \neq i$. Then we have $\boldsymbol{p}^{\prime} \in \mathcal{N}_{\mu}^{*}(\lambda)$, and $C(\boldsymbol{p})$ is obtained from an elementary excitation from $C\left(\boldsymbol{p}^{\prime}\right)$. By the hypothesis of induction we have $C\left(\boldsymbol{p}^{\prime}\right) \in \mathcal{E}_{\mu}^{*}(\lambda)$. So we have $C(\boldsymbol{p}) \in \mathcal{E}_{\mu}^{*}(\lambda)$.
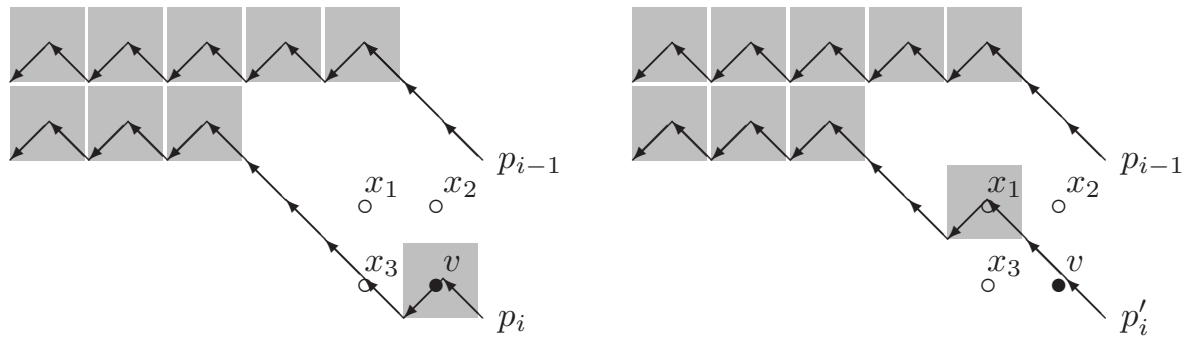

Next we consider the case that $v$ is on the diagonal. It is enough to consider type $I I$ case. Put $x_{1}=v-(1,1), x_{2}=v-(2,2), x_{3}=v-(1,0), x_{4}=v-(2,1)$.
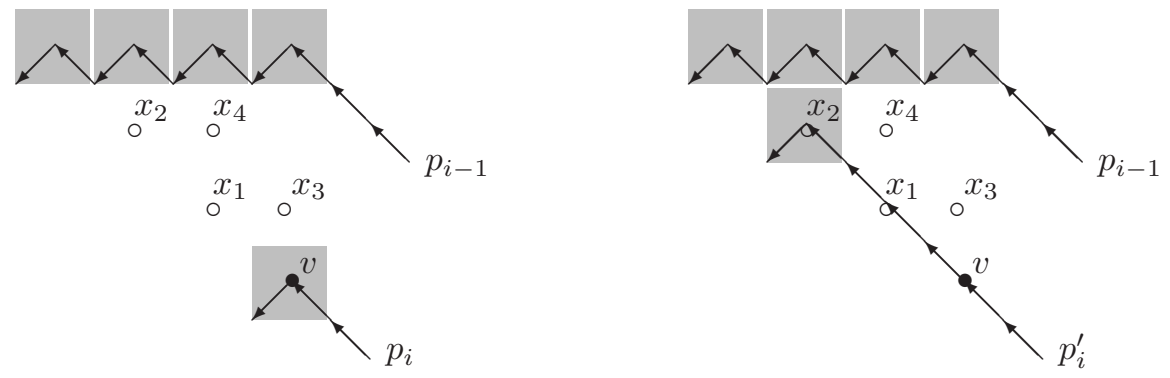

There are no paths going through either $x_{1}$ or $x_{2}$ since we have the restriction of the end point of the paths. Next we claim that $x_{3}, x_{4}$ do not belong to $C(\boldsymbol{p})$. Suppose $x_{k} \in C\left(p_{i-1}\right)$ for $k=3$ or 4 ; then the path $p_{i-1}$ must go through $x_{1}$ or $x_{2}$. This contradicts the above assertion. Thus in particular $x_{k}$ for $1 \leq k \leq 4$ are not in $C(\boldsymbol{p})$. Now we deform the path $p_{i}$ to $p_{i}^{\prime}$ as indicated in the figure and set $\boldsymbol{p}^{\prime}=\left(p_{1}^{\prime}, \ldots, p_{r}^{\prime}\right)$ with $p_{j}^{\prime}=p_{j}$ for $j \neq i$. Then by the same argument as in the preceding case, we have $C(\boldsymbol{p}) \in \mathcal{E}_{\mu}^{I I}(\lambda)$.

We shall describe the inverse map of $\boldsymbol{p} \mapsto C(\boldsymbol{p})$. Let $C$ be an element of $\mathcal{E}_{\mu}^{*}(\lambda)$. For each $(i, j) \in C$, we define its layer number as follows. Let $L_{m}$ denote the diagonal line $\left\{(i, j) \in \mathbb{Z}^{2} \mid j-i=m\right\}$. If $(i, j) \in L_{m}$ and

$$
C \cap L_{m}=\left\{\left(i_{1}, j_{1}\right), \ldots,\left(i_{s}, j_{s}\right)\right\}
$$

with $i_{1}<\cdots<i_{s}$, and $(i, j)=\left(i_{k}, j_{k}\right)$, then we define the layer number of $(i, j)$ to be $k$. Let $b_{1}, \ldots, b_{m} \in D_{\mu}^{\prime}$ be the boxes in $C$ with the layer number $k$. It is obvious 
that there exists a unique path starting from $u_{k}$ and going through all the points $b_{1}, \ldots, b_{m}$. Let us denote the path by $p_{k}$. Then form an $r$-tuple $\boldsymbol{p}_{C}=\left(p_{1}, \ldots, p_{r}\right)$.

Lemma 10.4. $\boldsymbol{p}_{C}$ is non-intersecting.

Proof. Clear from the construction.

Obviously if $C \in \mathcal{E}_{\mu}^{I I}(\lambda)$, then $\boldsymbol{p}_{C} \in \mathcal{N}_{\mu}^{I I}(\lambda)$. For any subset $C$ of $D_{\mu}^{\prime}$, we define its weight by $w(C)=\prod_{(i, j) \in C} a_{i j}$.

Proposition 10.5. Let $\lambda \leq \mu$ be strict partitions. There exists a weight preserving bijection between $\mathcal{N}_{\mu}^{I}(\lambda)$ and $\mathcal{E}_{\mu}^{I}(\lambda)$. Moreover, this map induces a weight preserving bijection between $\mathcal{N}_{\mu}^{I I}(\lambda)$ and $\mathcal{E}_{\mu}^{I I}(\lambda)$. Thus we have

$$
\sum_{C \in \mathcal{E}_{\mu}^{*}(\lambda)} w(C)=\sum_{\boldsymbol{p} \in \mathcal{N}_{\mu}^{*}(\lambda)} w(\boldsymbol{p}),
$$

for $*=I, I I$.

Let $w \leq v \in W^{P}$ and $\lambda \leq \mu \in \mathcal{S P}_{\rho_{n}}$ be the corresponding strict partitions. Then our combinatorial formula (Theorem 6.4) reads

$$
\left.\left[X_{w}\right]\right|_{v}=\sum_{C \in \mathcal{E}_{\mu}^{*}(\lambda)} w(C),
$$

where we choose the weights $a_{i j}=\beta_{i, j}$ which are given in Lemma 7.5. Now from Propositions 10.2 and 10.5 we have

$$
\left.\left[X_{\lambda}\right]\right|_{\mu}=\operatorname{Pf}\left(\left.\left[X_{\lambda_{i}, \lambda_{j}}\right]\right|_{\mu}\right),
$$

which is equal to $\left.\left(\operatorname{Pf}\left[X_{\lambda_{i}, \lambda_{j}}\right]\right)\right|_{\mu}$ since the map $\left.\alpha \mapsto \alpha\right|_{v}$ is a ring homomorphism. By virtue of the injectivity of the localization map, we have the equivariant Giambelli formula.

\section{ACKNOWLEDGMEnts}

We benefited from conversations with the participants in the "Workshop on Contemporary Schubert calculus and Schubert geometry" held at Banff in Canada, March 19-24, 2007. We thank the organizers of the workshop, Jim Carrell and Frank Sottile, for their kind invitation. We thank Sara Billey, Allen Knutson, Alexander Yong, Harry Tamvakis, Leonardo Mihalcea, Mark Shimozono, Soichi Okada, and Masao Ishikawa for valuable discussions.

The first author was partially supported by Grant-in-Aids for Young Scientists (B) (No. 17740101) from the Japan Society of the Promotion of Science.

\section{REFERENCES}

1. H. H. Andersen, J. C. Jantzen, and W. Soergel, Representations of quantum groups at a $p$ th root of unity and of semisimple groups in characteristic $p$ : independence of $p$, Asterisque No. 220 (1994), 321 pp. MR1272539(95j:20036)

2. A. Arabia, Cycles de Schubert et cohomologie équivariante de $K / T$, Invent. Math. 85 (1986), 39-52. MR842047 (87g:32036)

3. N. Bergeron and S. Billey, RC-graphs and Schubert polynomials, Exparemental Math. 2 (1993), no. 4, 257-269. MR1281474 (95g:05107) 
4. S. Billey, Kostant polynomials and the cohomology ring for $G / B$, Duke Math. J. 96 (1999), no. 1, 205-224. MR 1663931 (2000a:14060)

5. S. Billey and V. Lakshmibai, Singular loci of Schubert varieties, Progress in Mathematics, 182. Birkhäuser Boston, Inc., Boston, MA, 2000. xii+251 pp. ISBN: 0-8176-4092-4 MR1782635 (2001j:14065)

6. N. Bourbaki, Groupes et algèbres de Lie, Ch. IV, V, VI, Hermann, Paris, 1968. MR0240238 $(39: 1590)$

7. S. Fomin and A. Kirillov, The Yang-Baxter equation, symmetric functions, and Schubert polynomials, Discrete Math. 153 (1996), 123-143. MR1394950 (98b:05101)

8. S. R. Ghorpade and K. N. Raghavan, Hilbert functions of points on Schubert varieties in the symplectic Grassmannian, Trans. Amer. Math. Soc. 358 (2006), no. 12, 5401-5423 (electronic). MR 2238920 (2007d:14088)

9. W. Graham, Positivity in equivariant Schubert calculus, Duke Math. J. 109 (2001), no. 3, 599-614. MR.1853356 (2002h:14083)

10. T. Ikeda, Schubert classes in the equivariant cohomology of the Lagrangian Grassmannian, Adv. Math. 215 (2007), 1-23. MR2354984

11. V. N. Ivanov, Interpolation analogues of Schur $Q$-functions, Zap. Nauchn. Sem. S.-Peterburg. Otdel. Mat. Inst. Steklov. (POMI) 307 (2004), Teor. Predst. Din. Sist. Komb. i Algoritm. Metody. 10, 99-119, 281-282; translation in J. Math. Sci. (N. Y.) 131 (2005), no. 2, 54955507. MR2050689 (2004m:05268)

12. V. Kodiyalam and K. N. Raghavan, Hilbert functions of points on Schubert varieties in Grassmannians, Journal of Alg. 270 (2003), 28-54. MR2015929(2005d:14067)

13. A. Knutson and E. Miller, Gröbner geometry of Schubert polynomials, Ann. Math. 161 (2005), no. 3, 1245-1318. MR2180402(2006i:05177)

14. A. Knutson, E. Miller, and A. Yong, Gröbner geometry of vertex decompositions and flagged tableaux, preprint arXiv: math.AG/0502144

15. A. Knutson and T. Tao, Puzzles and (equivariant) cohomology of Grassmannians, Duke Math. J. 119 (2003), no. 2, 221-260. MR1997946 (2006a:14088)

16. B. Kostant and S. Kumar, The nil Hecke ring and cohomology of $G / P$ for a Kac-Moody group G, Adv. in Math. 62 (1986) 187-237. MR866159(88b:17025b)

17. V. Kreiman, Schubert classes in the equivariant K-theory and equivariant cohomology of the Grassmannian, preprint math.AG/0512204

18. V. Kreiman, Schubert classes in the equivariant K-theory and equivariant cohomology of the Lagrangian Grassmannian, preprint math.AG/0602245

19. V. Kreiman and V. Lakshmibai, Multiplicities of singular points in Schubert varieties of Grassmannians. Algebra, arithmetic and geometry with applications (West Lafayette, IN, 2000), 553-563, Springer, Berlin, 2004. MR2037109 (2005c:14060)

20. A. Kresch and H. Tamvakis, Double Schubert polynomials and degeneracy loci for the classical groups, Annales de l'institut Fourier, 52 (2002), no. 6, 1681-1727. MR1952528(2004d:14078)

21. S. Kumar, Kac-Moody groups, their flag varieties and representation theory, Progress in Math. 204, Birkhäuser, Boston, 2002. MR1923198 (2003k:22022)

22. V. Lakshmibai, K. N. Raghavan, and P. Sankaran, Equivariant Giambelli and determinantal restriction formulas for the Grassmannian, in: Special issue: In honor of Robert MacPherson, Part 1 of 3, Pure Appl. Math. Quart. 2 (3) (2006) 699-717. MR2252114 (2007h:14084)

23. V. Lakshmibai and J. Weyman, Multiplicities of points on a Schubert variety in a minuscule $G / P$, Adv. Math. 84 (1990), no. 2, 179-208. MR.1080976 (92a:14058)

24. A. Lascoux and M.-P. Schützenberger, Polynômes de Schubert, C. R. Acad. Sci. Paris, 294 (1982), 447-450. MR660739 (83e:14039)

25. A. Molev and B. E. A. Sagan, A Littlewood-Richardson rule for factorial Schur functions, Trans. Amer. Math. Soc. 351 (1999), no. 11, 4429-4443. MR1621694(2000a:05212)

26. P. Pragacz, Algebro-geometric applications of Schur $S$ - and $Q$-polynomials, Topics in invariant theory (Paris, 1989/1990), 130-191, Lecture Notes in Math., 1478, Springer, Berlin, 1991. MR 1180989 (93h:05170)

27. W. Rossmann, Equivariant multiplicities on complex varieties, Orbites unipotentes et représentations, III, Astérisque No. 173-174, (1989), 11, 313-330. MR.1021516 (91g:32042)

28. K. N. Raghavan and S. Upadhyay, Hilbert functions of points on Schubert varieties in orthogonal Grassmannians, preprint arXiv: 0704.0542 [math.CO] 
29. J. R. Stembridge, Nonintersecting paths, Pfaffians, and plane partitions, Adv. Math. 83 (1990), no. 1, 96-131. MR1069389(91h:05014)

30. J. R. Stembridge, On the fully commutative elements of Coxeter groups, J. Algebraic Combin. 5 (1996), no. 4, 353-385. MR1406459 (97g:20046)

Department of Applied Mathematics, Okayama University of Science, Okayama 7000005, JAPAN

E-mail address: ike@xmath.ous.ac.jp

Graduate School of Education, Okayama University, Okayama 700-8530, Japan

E-mail address: rdcv1654@cc.okayama-u.ac.jp 